\title{
The hepatokine Tsukushi is released in response to NAFLD and impacts cholesterol homeostasis
}

Mathilde Mouchiroud, ${ }^{1}$ Étienne Camiré, ${ }^{1}$ Manal Aldow, ${ }^{1}$ Alexandre Caron, ${ }^{2}$ Éric Jubinville, ${ }^{1}$ Laurie Turcotte, ${ }^{1}$ Inès Kaci, ${ }^{1}$ Marie-Josée Beaulieu, ${ }^{1}$ Christian Roy, ${ }^{1}$ Sébastien M. Labbé, ${ }^{1,3}$ Thibault V. Varin,, ${ }^{1,4}$ Yves Gélinas, ${ }^{1}$ Jennifer Lamothe, ${ }^{1}$ Jocelyn Trottier, ${ }^{5,6}$ Patricia L. Mitchell, Frédéric Guénard, ${ }^{4}$ William T. Festuccia, ${ }^{7}$ Philippe Joubert, ${ }^{1}$ Christopher F. Rose, ${ }^{8}$ Constantine J. Karvellas, ${ }^{9}$ Olivier Barbier, ${ }^{5,6}$ Mathieu C. Morissette, ${ }^{1,10}$ André Marette, ${ }^{1,4,10}$ and Mathieu Laplante ${ }^{1,10,11}$

'Centre de Recherche de l'Institut Universitaire de Cardiologie et de Pneumologie de Québec - Université Laval (CRIUCPQ), Québec City, Québec, Canada. 'Division of Hypothalamic Research, Department of Internal Medicine, University of Texas Southwestern Medical Center, Dallas, Texas, USA. ${ }^{3}$ IPS Thérapeutique, Sherbrooke, Québec, Canada. ${ }^{4}$ Institut sur la Nutrition et les Aliments Fonctionnels (INAF), Université Laval, Québec City, Québec, Canada. ${ }^{5}$ Laboratory of Molecular Pharmacology, Endocrinology-Nephrology Axis, Centre de Recherche du Centre Hospitalier Universitaire de Québec, Québec City, Québec, Canada. ${ }^{6}$ Faculty of Pharmacy, Université Laval, Québec City, Québec, Canada. 'Department of Physiology and Biophysics, Institute of Biomedical Sciences, University of São Paulo, São Paulo, Brazil. ${ }^{8}$ Hepato-Neuro Laboratory, Centre Hospitalier de l'Université de Montréal (CRCHUM), Montréal, Québec, Canada. 'Liver Unit, Division of Gastroenterology, Department of Critical Care Medicine, School of Public Health Science, University of Alberta, Edmonton, Alberta, Canada. ${ }^{10}$ Département de Médecine and ${ }^{11}$ Centre de Recherche sur le Cancer de I'Université Laval, Université Laval, Québec City, Québec, Canada.

Nonalcoholic fatty liver disease (NAFLD) prevails in obesity and is linked to several health complications including dyslipidemia and atherosclerosis. How exactly NAFLD induces atherogenic dyslipidemia to promote cardiovascular diseases is still elusive. Here, we identify Tsukushi (TSK) as a hepatokine induced in response to NAFLD. We show that both endoplasmic reticulum stress and inflammation promote the expression and release of TSK in mice. In humans, hepatic TSK expression is also associated with steatosis, and its circulating levels are markedly increased in patients suffering from acetaminophen-induced acute liver failure (ALF), a condition linked to severe hepatic inflammation. In these patients, elevated blood TSK levels were associated with decreased transplant-free survival at hospital discharge, suggesting that TSK could have a prognostic significance. Gain- and loss-of-function studies in mice revealed that TSK impacts systemic cholesterol homeostasis. TSK reduces circulating HDL cholesterol, lowers cholesterol efflux capacity, and decreases cholesterol-to-bile acid conversion in the liver. Our data identify the hepatokine TSK as a blood biomarker of liver stress that could link NAFLD to the development of atherogenic dyslipidemia and atherosclerosis.

Conflict of interest: The authors have declared that no conflict of interest exists.

Copyright: () 2019, American Society for Clinical Investigation.

Submitted: April 29, 2019

Accepted: June 27, 2019

Published: August 8, 2019.

Reference information: /CI Insight. 2019:4(15):e129492.

https://doi.org/10.1172/jici.

insight.129492.

\section{Introduction}

Obesity has reached epidemic proportions worldwide (1-3). Excessive accumulation of white adipose tissue, especially in the abdominal cavity, is a major risk factor for several diseases including type 2 diabetes, insulin resistance, dyslipidemias, hypertension, and cardiovascular diseases $(4,5)$. Abdominal obesity is also tightly linked to the development of nonalcoholic fatty liver disease (NAFLD), a wide spectrum of liver conditions that ranges from simple steatosis to nonalcoholic steatohepatitis (NASH), a disease characterized by hepatic inflammation and fibrosis that can progress to cirrhosis and hepatocellular carcinoma $(6,7)$. NAFLD is one of the most important causes of liver disease worldwide, and its prevalence is constantly rising $(8,9)$.

In addition to its direct impact on liver health, NAFLD is suspected to play an active role in the development of obesity-related metabolic disorders. Links between NAFLD and dyslipidemias, glucose intolerance, insulin resistance, type 2 diabetes, and cardiovascular diseases have been reported (10-14). 
In fact, cardiovascular complications represent the leading cause of death in patients with NAFLD (15). How exactly NAFLD contributes to these diseases is unclear. Recent advances suggest that the liver may impact metabolism through the secretion of specific proteins. These proteins, often termed hepatokines, are secreted by the liver and mediate interorgan communication through autocrine, paracrine, and endocrine signaling $(8,16)$. Several hepatokines have been identified including fetuin A (17), fetuin B (18), fibroblast growth factor 21 (FGF21) (19), fibrinogen-like protein 1 (FGL1, also known as hepassocin) (20), follistatin (FST) (21-23), inhibin $\beta E$ (INHBE) $(24,25)$, leukocyte cell-derived chemotaxin 2 (LECT2) (26), retinol-binding protein 4 (RBP4) $(27,28)$, and selenoprotein P (SEPP1) (29). These hepatokines, whose expression is affected by conditions such as obesity and NAFLD, impact various organs to modulate glucose metabolism, lipid homeostasis, and inflammation. Studies in mice and humans indicate that altered hepatokine secretion promotes glucose intolerance, insulin resistance, NAFLD progression, and cardiovascular diseases $(8,16)$, making these proteins attractive targets to alleviate obesity-related metabolic disorders.

The list of hepatokines affecting metabolism has expanded in recent years. Nevertheless, because of the importance of the liver in the regulation of systemic homeostasis, it is likely that this organ communicates its state through effectors that are not yet characterized $(18,30)$. Here, using a comprehensive analytical platform, we report the identification of TSK as a protein produced and secreted by the liver. We find that hepatic Tsk expression and circulating levels are elevated in various obese mouse models and strongly induced in response to lipid deposition in the liver. The presence of endoplasmic reticulum (ER) stress and inflammation, both common in NAFLD, promotes the expression and release of TSK. In humans, hepatic TSK expression correlates with steatosis, and high plasma TSK levels were found in patients suffering from acetaminophen-induced acute liver failure (ALF), a condition linked to severe inflammation and liver damage. In these patients, elevated TSK levels in serum were associated with decreased transplant-free survival at hospital discharge. Studies in mice revealed that TSK does not affect NAFLD development and progression but rather plays a role in the regulation of systemic cholesterol homeostasis. Here, we report that TSK lowers circulating HDL-cholesterol levels, reduces cholesterol efflux capacity, and decreases cholesterol conversion to bile acids (BAs) in the liver. We propose a model in which TSK is released in response to NAFLD to limit reverse cholesterol transport. Our data identify the hepatokine TSK as a blood biomarker of liver stress that could link NAFLD to the development of atherogenic dyslipidemia and atherosclerosis.

\section{Results}

TSK is a hepatokine induced by obesity. In order to identify secreted factors primarily produced by the liver whose expression is deregulated in obesity, we have implemented a simple analytical platform using BioGPS (http://biogps.org), a centralized gene-annotation portal that enables researchers to access distributed gene annotation resources $(31,32)$. BioGPS hosts a reference plugin that displays gene expression patterns from more than 90 mouse tissues and cell types (33). These microarray data, available through NCBI's Gene Expression Omnibus (GEO accession number GSE10246), can be used to compare the expression profile of any given gene between a broad panel of tissues. To establish a list of liver-specific genes, we first interrogated the database to extract all the genes showing an expression pattern correlating with the albumin-encoding gene, which is selectively expressed in hepatocytes (Figure 1A). The correlation cutoff was arbitrarily fixed at 0.80 . This led to the identification of 306 liver-specific genes (Supplemental Table 1; supplemental material available online with this article; https://doi.org/10.1172/jci.insight.129492DS1), including several established hepatokines. All the genes were then scored to sort out the best candidates. Because we were interested in secreted proteins, priority was given to genes coding for proteins with a secretory signal peptide. Conserved proteins with uncharacterized relation to obesity were also prioritized. This analysis led to the identification of 17 high-priority candidates (Supplemental Table 2). To identify which of these genes are affected by obesity, we next measured their expression in the liver of lean and obese $(d b / d b)$ mice by quantitative PCR (qPCR). Of the 17 candidates, 7 were differentially expressed between lean and obese mice, namely Cpn1, Gdf2, Habp2, Inhbc, Mia2, Tsk, and Vwce (Supplemental Figure 1A).

$T s k$ is one candidate gene that caught our attention. As shown in Figure 1B, its expression is specifically elevated in mouse liver. Our analysis revealed that hepatic Tsk was the candidate gene showing the greatest difference in relative expression between lean and obese mice (Supplemental Figure 1A). Specifically, Tsk 
A

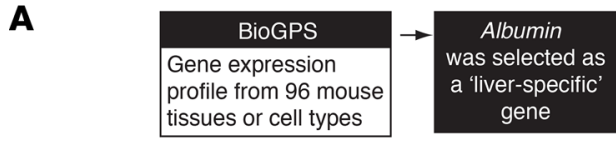

B

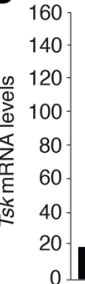

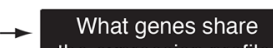

the expression profile

of Albumin?

cutoff $>0.80$

- 306 genes identified
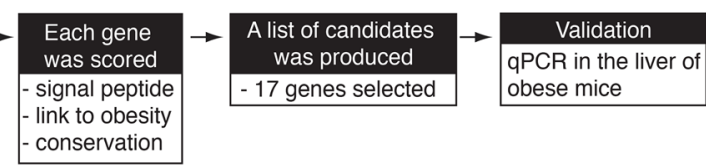

conservation

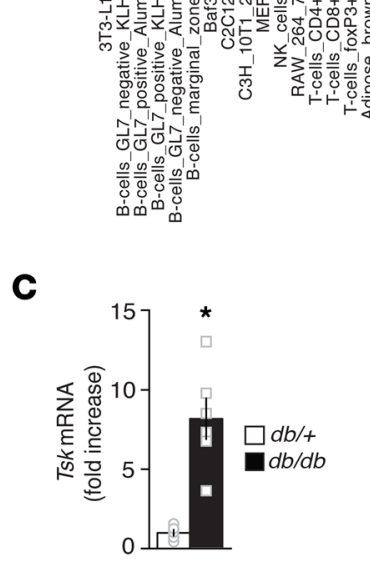

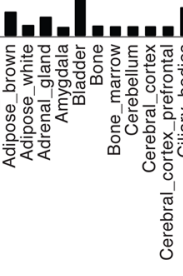

D

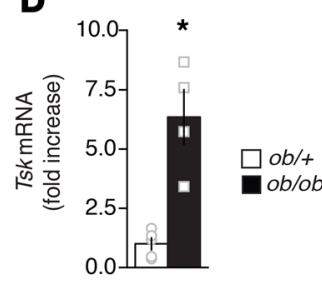

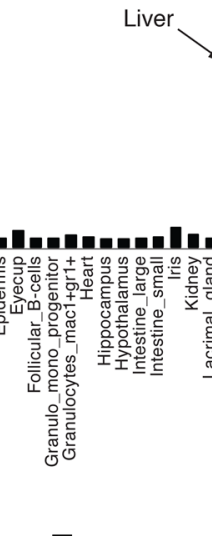

E

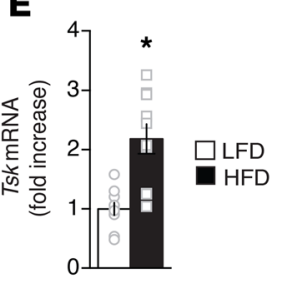

G

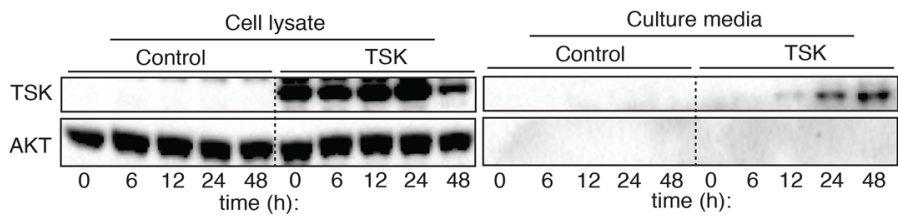

I

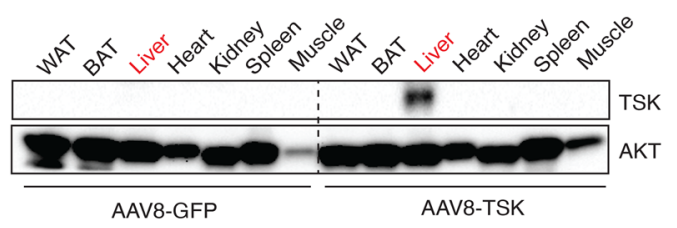

J

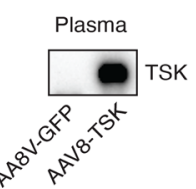

F

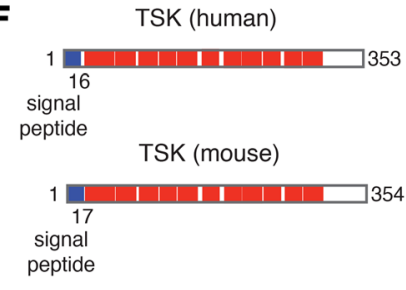

H
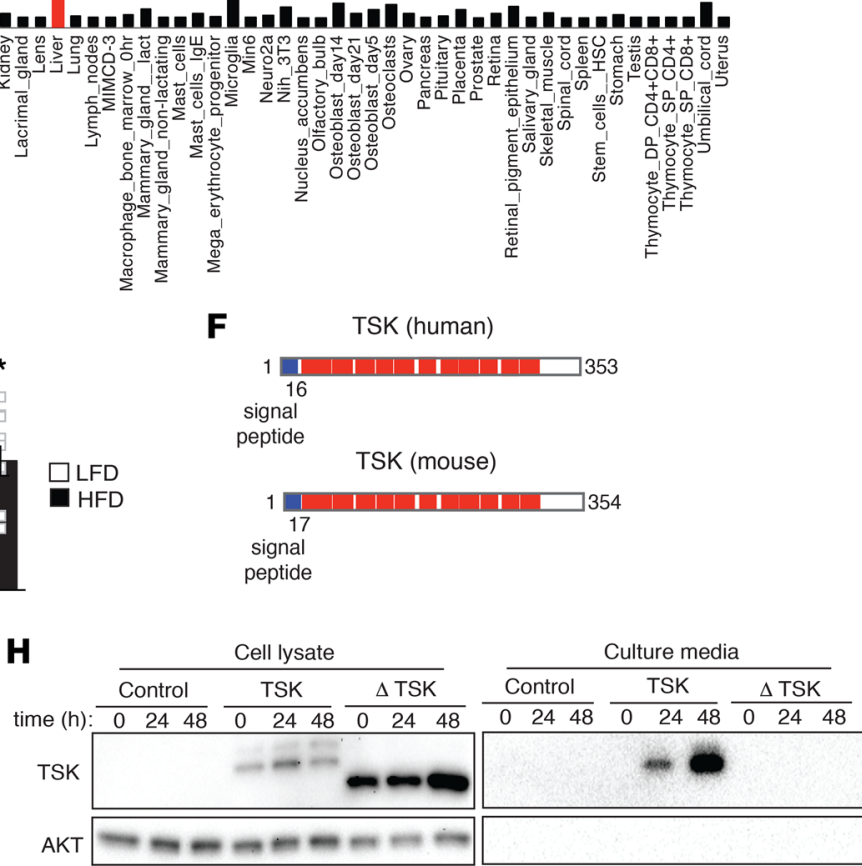

$\mathbf{K}$

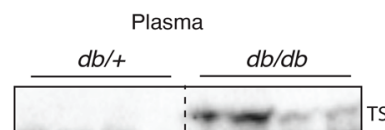

$\mathbf{L}$

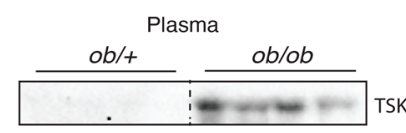

Figure 1. TSK is a hepatokine induced by obesity. (A) Summary of the experimental approach designed to identify liver-specific secreted proteins regulated in conditions of obesity. (B) Gene expression profile comparing the expression of Tsk in various mouse tissues and cell types. The microarray data used in this panel are available through NCBI's Gene Expression Omnibus (accession number CSE10246). (C-E) qPCR analysis of Tsk transcript expression in the liver of (C) littermate control and $d b / d b$ mice ( $n=5-6 /$ group) (10 weeks old), (D) littermate control and ob/ob mice ( $n=4-5 / g r o u p)$ (12 weeks old), and (E) LFD- and HFD-fed C57BL/6) mice ( $n=10 /$ group) (11-13 weeks old fed LFD or HFD for 10 weeks). Data represent the mean \pm SEM. Significance was determined by 2-tailed, unpaired $t$ test. ${ }^{*} P<0.05$ versus controls. (F) Schematic representation of human and mouse TSK protein. (C) Western blot analysis of cell lysates and culture media of HEK 293T cells overexpressing human TSK. Cells were plated the day before and culture medium was changed at time 0 . Cells were lysed and culture medium was collected at the indicated times. AKT was used as a loading control. (H) Western blot analysis of cell lysates and culture media of HEK 293T cells overexpressing human TSK or a mutated form lacking the signal peptide ( $\triangle$-TSK). Samples were processed as described in G. (I and J) Western blot analysis of (I) tissues samples or (J) plasma collected from [57BL/6) mice injected with AAV8-GFP or AAV8-TSK. Mice were sacrificed 4 weeks following AAV8 injection. (K and L) Western blot analysis of plasma TSK levels in (K) control and $d b / d b$ mice and (L) control and $o b / o b$ mice. Representative samples are shown.

expression was induced more than 8 times in the liver of $d b / d b$ mice (Figure $1 C$ ). Following up on this finding, we found that Tsk expression was also significantly increased in the liver of other obese mouse models such as $o b / o b$ mice and mice fed a high-fat diet (HFD) (Figure 1, D and E). Of note, we observed that the elevation in Tsk expression was not as profound in HFD-fed mice compared with genetically obese mice, suggesting that the severity of obesity may be an important factor in the regulation of Tsk expression (Supplemental Figure 1, B-D). Despite these differences, our results identify Tsk as a gene primarily expressed in the liver whose expression is induced in response to obesity. 
TSK is a secreted protein enriched in the plasma of severely obese mice. TSK is a protein that carries an N-terminal signal peptide and 10 leucine-rich repeat (LRR) domains in its central region (Figure $1 F)$. This protein is conserved throughout evolution, with homologs found from human to zebrafish (Supplemental Figure 1E). TSK is an atypical member of the small leucine-rich proteoglycan (SLRP) family, a group of 18 proteins that bind to components of the extracellular matrix to modulate the action of several signaling pathways (34). TSK was shown to control developmental processes by modulating bone morphogenetic protein (BMP) (35-38), Wnt (39), FGF (37), and TGF- $\beta$ signaling pathways $(40,41)$.

The presence of a signal peptide in the N-terminal section of TSK indicates that the protein may be secreted. Studies performed in chick and Xenopus support this possibility $(35,36,38)$. To directly test whether mammalian TSK is also secreted, TSK was overexpressed in HEK 293T cells and culture medium was collected for Western blot analysis (Figure 1G). TSK was detected in the culture media of overexpressing cells, thus confirming the secreted nature of the protein. Mutated TSK lacking the signal peptide $(\Delta$-TSK) could not be secreted in the same system, indicating the importance of this domain for the release of the protein in the extracellular compartment (Figure $1 \mathrm{H}$ ). To directly test the ability of the liver to secrete TSK in vivo, mice were infected with adeno-associated virus serotype 8 (AAV8) coding for either a control green fluorescent protein (GFP) or full-length TSK. As expected, we detected high expression of TSK in the liver of mice injected with AAV8-TSK (Figure 1I and Supplemental Figure 1F). Importantly, we also found high levels of the protein in the plasma, thus confirming the ability of the liver to produce and secrete TSK in the circulation (Figure 1J).

As discussed above, Tsk is highly expressed in the liver and its expression is induced in obese mice. To determine whether such increase translates into a rise in circulating TSK, plasma collected from lean and obese mice was analyzed by Western blotting. Circulating TSK levels were barely detectable in lean animals but were high in genetically obese mice (Figure 1, K and L). Despite those striking results observed in severely obese mice, TSK could not be detected in the plasma of HFD-fed animals (data not shown). As shown above, the rise in hepatic Tsk expression was less severe in HFD-fed mice compared with genetically obese animals (Figure 1, C-E), suggesting that a certain threshold of hepatic TSK production may have to be reached to allow the detection of the protein in the blood using our antibody. Nevertheless, we can conclude from these results that TSK is a secreted protein that circulates at high levels in severely obese animals.

Hepatic Tsk expression correlates with NAFLD in multiple mouse models. NAFLD is a condition that prevails in obesity (9). To define whether NAFLD impacts Tsk expression, hepatic triglyceride levels were measured in lean and obese mice and correlations with Tsk mRNA were calculated. As shown in Figure 2, A-C, we observed a strong positive relationship between liver triglyceride content and Tsk expression in all the models tested, suggesting a prominent role of liver fat accumulation in promoting Tsk expression.

We next examined whether NAFLD per se could, independently of obesity, promote Tsk expression and secretion. Two different approaches were used to test this hypothesis. We first fed mice a methionineand choline-deficient (MCD) diet for 21 days. This approach induced liver steatosis despite significant weight loss (Figure 2, D and E). As observed in obese mice, we found an elevation in both hepatic Tsk expression and circulating TSK levels in mice fed the MCD diet (Figure 2, F and G). A positive correlation was also observed between liver triglycerides and Tsk mRNA (Figure 2H). Interestingly, switching back MCD diet-fed mice to a normal diet for 3, 6, or 12 days was sufficient to reverse steatosis and normalize hepatic Tsk expression and secretion (Figure 2, I-L). Again, a positive link between liver triglycerides and Tsk mRNA was found in this experiment (Supplemental Figure 2A). In a second approach, mice were treated with the synthetic liver X receptor (LXR) agonist T0901317 for 4 days to induce acute liver steatosis without weight gain, as shown previously (42). In this model, we also found a clear positive association between liver triglycerides and Tsk expression (Supplemental Figure 2, B-D). These results indicate that NAFLD, independently of obesity, promotes hepatic Tsk expression and secretion.

Recently, Wang et al. reported that hepatic Tsk expression and secretion are highly induced in response to cold exposure and injection with the $\beta 3$ adrenergic receptor agonist CL316243 (43). These observations were confirmed here (Figure 2M). It is well known that cold and CL316243 strongly stimulate lipolysis in adipose tissue in order to support thermogenesis (44). As expected from these results, we measured a significant increase in circulating nonesterified free fatty acids (NEFAs) (Supplemental Figure 2E) and hepatic triglycerides (Figure 2N) in response to cold and CL316243. As for all the other mouse models described above, a positive correlation between liver triglycerides and Tsk expression was observed, suggesting that 
A

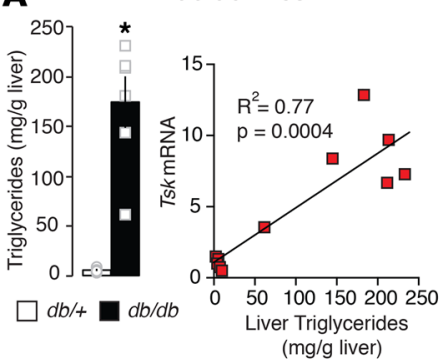

B

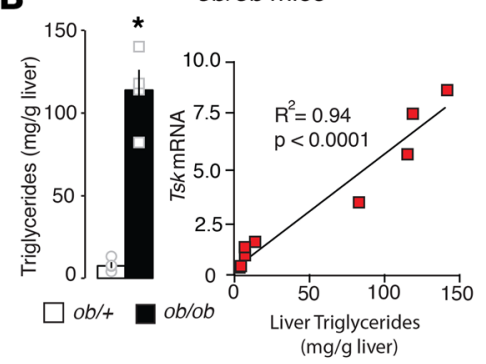

D

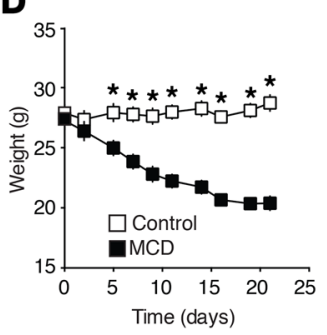

$\mathbf{E}^{100} 7$ *
$\mathbf{F}$

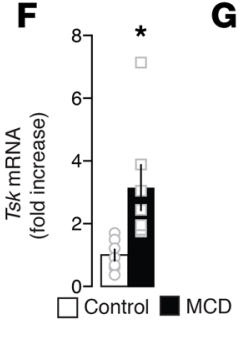

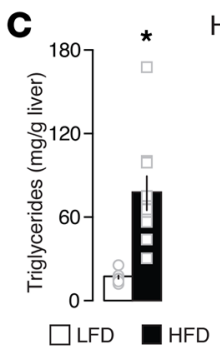

HFD-fed mice

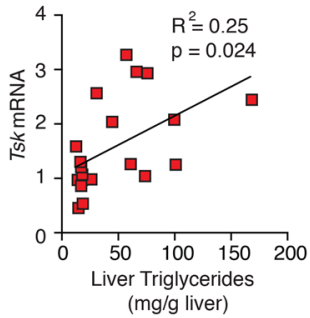

H
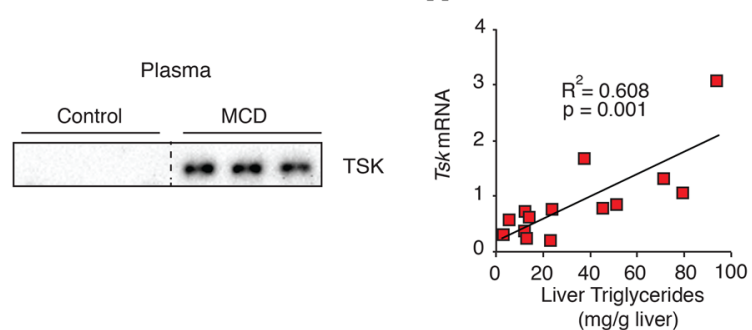

I

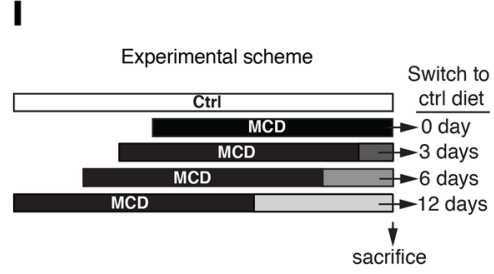

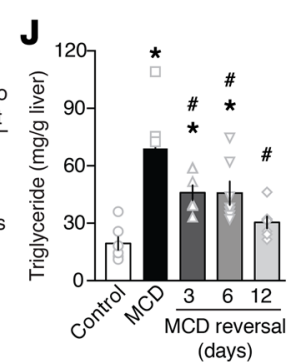

$\mathbf{K}^{4} \quad \mathbf{L}$

L $\quad$ Plasma
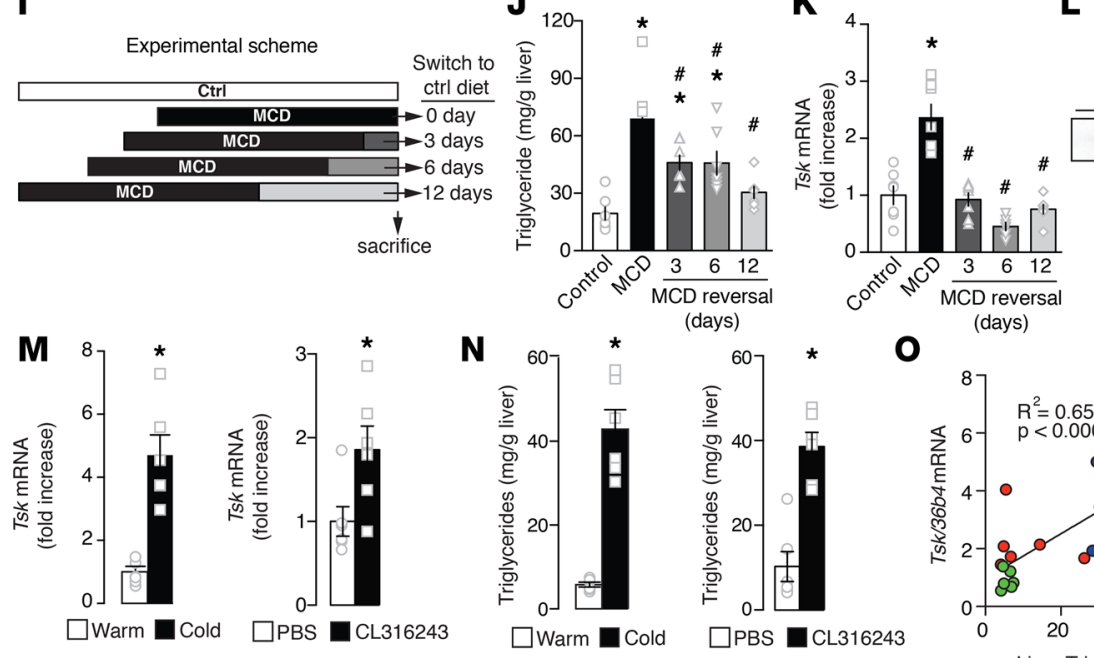

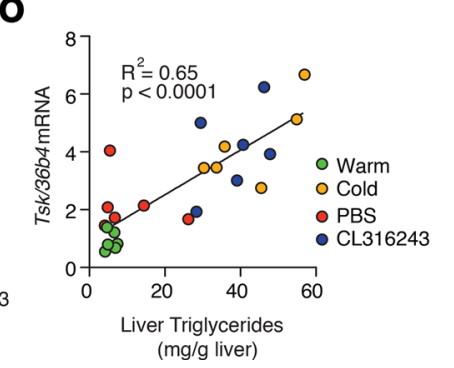

Figure 2. Tsk expression correlates with liver steatosis in multiple mouse models. (A-C) Hepatic triglyceride content in $(\mathbf{A})$ control and $d b / d b$ mice $(n=$ 5-6/group) (10 weeks old), (B) control and ob/ob mice ( $n=4-5 /$ group) (12 weeks old), or (C) LFD- and HFD-fed mice ( $n=10 /$ group) (11-13 weeks old fed LFD or HFD for 10 weeks). For each mouse model, the correlation between Tsk transcript levels and hepatic triglyceride content is presented. (D) Body weight of male mice fed a control or MCD diet for 21 days ( $n=7 /$ group). (E) Hepatic triglyceride content measured in mice fed a control or MCD diet ( $n=7 /$ group). (F) qPCR analysis of Tsk expression in the liver of control- and MCD diet-fed mice ( $n=7 /$ group). (C) Western blot analysis of plasma TSK levels in controland MCD diet-fed mice. Representative samples are shown. (H) Correlation calculated between Tsk transcript levels and hepatic triglyceride content in control- and MCD diet-fed mice $(n=14)$. (I) Experimental scheme of the MCD reversal experiments. Mice were fed a control or MCD diet for 21 days before being switched back to a control diet for 3, 6, or 12 days. (J) Hepatic triglyceride content and (K) Tsk mRNA expression levels measured in mice included in the MCD reversal study described in I ( $n=6-7 /$ group). (L) Western blot analysis of plasma TSK levels in the MCD reversal study. Representative samples are shown. (M) qPCR analysis of Tsk expression in the liver of male mice housed at thermoneutrality $\left(30^{\circ} \mathrm{C}\right)$ or exposed to cold $\left(10^{\circ} \mathrm{C}\right)$ for 6 hours $(n=6 /$ group) (left part) or in mice injected with saline (vehicle) or CL316243 ( $0.1 \mathrm{mg} / \mathrm{kg}$ ) for 4 hours ( $n=6 /$ group) (right panel). In these experiments, male mice (10-12 weeks old) were used. (N) Hepatic triglyceride content in the experiment described in $\mathbf{M}$. (0) Correlation calculated between Tsk transcript levels and hepatic triglyceride content in control mice and mice exposed to cold or injected with CL316243 as described in $\mathbf{M}(n=24)$. In all panels, data represent the mean \pm SEM. In A-F, M, and $\mathbf{N}$ significance was determined by 2-tailed, unpaired $t$ test. ${ }^{*} P<0.05$ versus controls. Pearson correlations (2-tailed) were calculated in A-C, H, and $\mathbf{O}$. In D, 2-way ANOVA with Sidak's multiple-comparisons test was performed. ${ }^{*} P<0.05$ versus control. In J and $\mathbf{K}, 1$-way ANOVA with Tukey's multiple-comparisons test was performed. ${ }^{*} P<0.05$ versus control. ${ }^{\#} P<0.05$ versus MCD.

hepatic lipid deposition plays a prominent role in promoting Tsk transcription in response to $\beta 3$ adrenergic receptor stimulation (Figure 2O).

$E R$ stress and inflammation promote Tsk transcription and release. Hepatic steatosis is often accompanied by ER stress and inflammation $(45,46)$. To ascertain whether these biological processes participate in the control 
of Tsk expression, a series of experiments were conducted. First, to determine whether ER stress plays a role in regulating Tsk expression, $78-\mathrm{kDa}$ glucose-regulated protein (Grp78) expression was measured in the liver of severely obese mice as a marker of the unfolded protein response (UPR), a signaling cascade activated by ER stress (47). Grp 78 mRNA expression was significantly increased in the liver of obese mice (Supplemental Figure 3A). In these animals, we observed a strong positive association between Grp 78 and Tsk expression (Supplemental Figure 3B). Elevated GRP78 levels were also measured in the liver of MCD diet-fed mice (Supplemental Figure 3C). To directly test the impact of ER stress on Tsk transcription, AML12 hepatocytes were treated with escalating doses of tunicamycin, a nucleoside antibiotic that blocks N-linked glycosylation and induces severe ER stress. We found that tunicamycin acutely increased Tsk expression in a dose-dependent manner (Figure 3A). The same effect was observed in the liver of mice acutely injected with tunicamycin (Figure 3B). Further supporting the role of the UPR in the regulation of Tsk expression, we found that the ER-stress-mediated increase in Tsk transcription was reduced upon inhibition of endoribonuclease/protein kinase IRE1-like protein (IRE1A) and protein kinase RNA-like endoplasmic reticulum kinase (PERK), two major effectors of the UPR (Figure 3C).

We next tested the contribution of inflammation to the regulation of hepatic Tsk expression. Elevated levels of proinflammatory cytokines were found in the liver of all the NAFLD mouse models tested (Supplemental Figure 3, D-G). To define the direct impact of inflammation on Tsk transcription, AML12 cells were treated with a cocktail of proinflammatory cytokines. Strikingly, we found that cytokines significantly increased Tsk expression (Figure 3D and Supplemental Figure $3 \mathrm{H}$ ). We next used carbon tetrachloride $\left(\mathrm{CCl}_{4}\right)$ to induce an acute liver injury and hepatic inflammation in mice. A single dose of $\mathrm{CCl}_{4}$ caused severe inflammation in the mice (Figure 3E). This increase was associated with elevated hepatic Tsk expression and high circulating TSK levels (Figure 3, F and G). We also found that lipopolysaccharide (LPS), a component of gram-negative bacteria with potent proinflammatory properties, increased circulating TSK levels in mice (Supplemental Figure 3I). These results indicate that inflammation drives Tsk expression. Altogether, our observations support the idea that lipid flux to the liver, ER stress, and inflammation probably play complementary roles in promoting Tsk expression in NAFLD.

TSK levels are increased in response to steatosis and liver damage in humans. Because Tsk expression was increased in response to NAFLD in mice, we next asked whether this was also observed in humans. Liver samples were collected from a cohort of obese patients undergoing bariatric surgery $(n=58)$. Triglycerides were extracted and patients were distributed into 2 groups based on the median liver triglyceride content (Figure 4, A and B). Interestingly, we found that TSK expression was significantly higher in patients with elevated hepatic triglycerides (Figure 4C), thus confirming that TSK expression is linked to NAFLD not only in mice but also in humans. In these samples, we found a significant association between inflammatory markers and TSK expression, reinforcing the possible role of inflammation in promoting TSK expression in humans (Supplemental Figure 4, A and B). As described above in HFD-fed mice, circulating TSK levels remained under the detection limit of our method in this human cohort (data not shown).

In order to demonstrate that TSK circulates at high levels in the presence of severe inflammation and hepatic ER stress in humans, we utilized samples collected from patients suffering from acetaminophen-induced ALF, a condition known to cause inflammation, impair ER function, and promote UPR activation (48, 49). Blood samples were collected from ALF patients rapidly after their admission to the intensive care unit and over the days following their admission (Supplemental Figure 4C). As shown in Figure 4, D and E, plasma TSK levels were markedly elevated in many patients tested upon admission (10 out of 26). In every case, plasma TSK levels went down at later time points. Interestingly, we observed that elevated TSK levels in serum were associated with decreased transplant-free survival at hospital discharge. As shown in Figure 4F, TSK was detected in serum samples of $87.5 \%$ of the patients ( 7 of 8 ) that either died or received a liver transplant versus only $16.6 \%$ of the patients that survived (3 of 18). Consistently, TSK protein levels were significantly higher in patients that died or received a liver transplant compared with the survivors (Figure 4G). Altogether, these results indicate that TSK is a hepatokine that circulates at high levels in response to severe hepatic stress, both in mice and humans, and that serum TSK might have prognostic significance in patients with ALF.

Modulating TSK expression does not affect NAFLD development. To define the functions of TSK in vivo, we injected mice with AAV8-TSK to overexpress TSK in the liver and elevate its circulating levels (Figure 1I, Figure 5, A and B, and Supplemental Figure 5A). Mice overexpressing TSK did not show visible physiological differences compared to control mice (Supplemental Figure 5B). Fasting glucose and insulin levels were not different between controls and AAV8-TSK-injected mice (Supplemental Figure 5, C and D). 
A
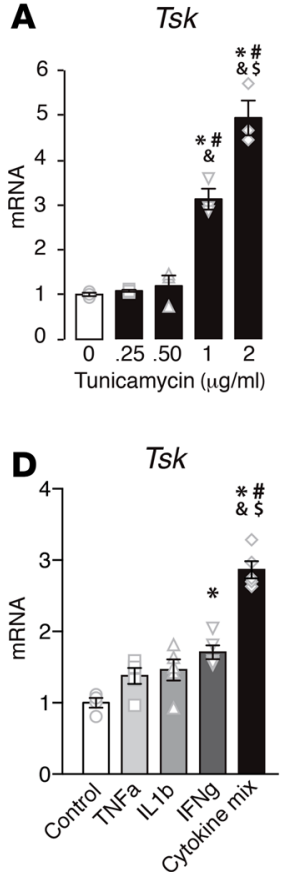

Grp78

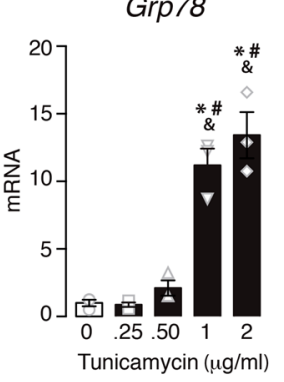

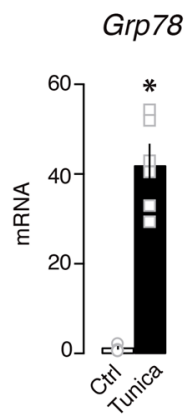

Grp78

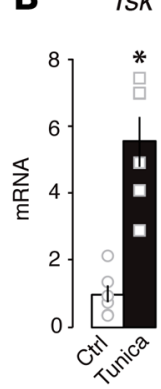

C

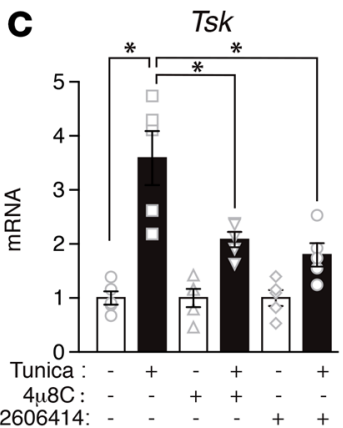

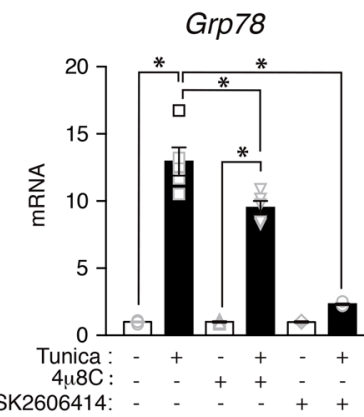

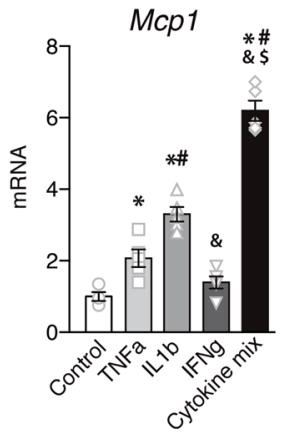

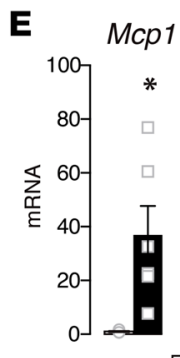

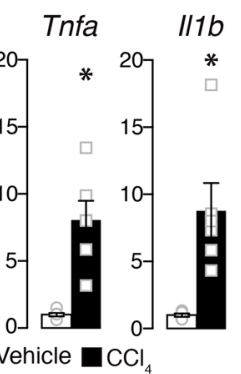

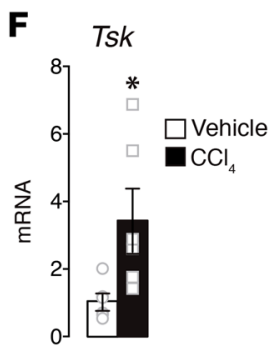

G

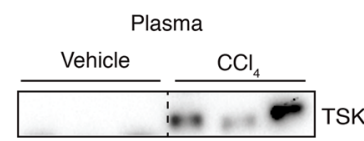

Figure 3. ER stress and inflammatory mediators promote Tsk expression and release. (A) qPCR analysis of Tsk and Grp78 transcript levels in AML12 hepatocytes treated or not with tunicamycin for 8 hours ( $n=3 /$ condition). (B) qPCR analysis of Tsk and Grp78 transcript levels in the liver of male mice ( 10 weeks old) injected with tunicamycin ( $n=6 /$ group). Mice were injected with tunicamycin ( $2 \mathrm{mg} / \mathrm{kg}$ ) and sacrificed 8 hours later. (C) qPCR analysis of Tsk and Grp78 transcript levels in AML12 cells pretreated with the indicated inhibitors $(4 \mu 8 \mathrm{C}, 100 \mu \mathrm{M}$; GSK2606414, $1 \mu \mathrm{M})$ for 1 hour and then treated or not with tunicamycin for 8 hours ( $n=5$ /condition). (D) qPCR analysis of Tsk and Mcp1 transcript levels in AML12 cells treated with TNF ( $5 \mathrm{ng} / \mathrm{mL})$, IL-1 $13(20 \mathrm{ng} / \mathrm{mL})$, IFN- $\gamma(5000 \mathrm{U} /$ $\mathrm{mL}$ ), or a mixture of these cytokines for 8 hours ( $n=5 /$ condition). (E) qPCR analysis of Mcp1, Tnfa, and I/1b transcripts in the liver of male mice (10-12 weeks old) injected with either vehicle or $\mathrm{CCl}_{4}(40 \%, 1 \mathrm{~mL} / \mathrm{kg})(n=6$ mice/group). Mice were sacrificed 24 hours after the injection. (F) qPCR analysis of Tsk transcript in the liver of mice injected with either vehicle or $\mathrm{CCl}_{4}$. (C) Western blot analysis of plasma TSK levels in mice injected with either vehicle or $\mathrm{CCl}_{4}$. Representative samples are shown. In all panels, data represent the mean \pm SEM. One-way ANOVA with Tukey's multiple-comparisons test was performed in $\mathbf{A}$, $\mathbf{C}$, and $\mathbf{D}$. In $\mathbf{A},{ }^{*} P<0.05$ versus dose $0 ;{ }^{\#} P<0.05$ versus dose $0.25 \mu \mathrm{g} / \mathrm{mL} ;{ }^{\&} P<0.05$ versus dose $0.50 \mu \mathrm{g} / \mathrm{mL} ;{ }^{\$} P<0.05$ versus dose $1.0 \mu \mathrm{g} / \mathrm{mL}$. In $\mathbf{C},{ }^{*} P<0.05$. In $\mathbf{D}$, ${ }^{*} P<$ 0.05 versus control; ${ }^{\#} P<0.05$ versus TNF- $\alpha$; ${ }^{\&} P<0.05$ versus IL-1 $\beta ;{ }^{\$} P<0.05$ versus IFN- $\gamma$. In $\mathbf{B}, \mathbf{E}$, and $\mathbf{F},{ }^{*} P<0.05$ versus controls by 2 -tailed, unpaired $t$ test.

We next looked at the liver histology and composition to define whether TSK could directly affect NAFLD development. As shown in Figure 5C, we found no evidence of steatosis in AAV8-TSK mice. Supporting these observations, the biochemical composition of the liver (water, triglyceride, protein, and glycogen) did not differ between the groups (Figure 5D). Gene expression analyses revealed that TSK did not promote inflammation, ER stress, and fibrosis (Figure 5E). Altogether, these results indicate that TSK per se does not promote NAFLD development in mice.

Studies were next performed with TSK-knockout (TSK-KO) mice (Figure 5, F-H). Because TSK levels are elevated in response to obesity and steatosis, experiments with TSK-KO mice were first performed in both lean and obese mice. TSK-null animals were smaller compared with wild-type littermates (Supplemental Figure 5E). This phenotype was reported previously and is consistent with the established role of TSK in regulating development in mice $(50,51)$. No differences in body weight gain were found between wild-type and TSK-null mice exposed to HFD (Supplemental Figure 5F). Consistently, we found no major differences in organ weight between wild-type and TSK-KO mice (Supplemental Figure 5G). As observed in mice overexpressing TSK, fasting glucose and insulin were not different between wildtype and TSK-null mice (Supplemental Figure 5, H and I). TSK loss did not affect liver fat accumulation (Figure 5I). The biochemical composition of the liver (water, triglyceride, protein, and glycogen) did not differ between the groups (Figure 5J). We observed mild signs of UPR activation in the liver of TSK-null mice but no impact on inflammation and fibrosis (Figure $5 \mathrm{~K}$ ). Additional studies performed using TSK wild-type and $-\mathrm{KO}$ mice bred to the $o b / o b$ background also failed to show a major impact of TSK loss on the severity and the progression of NAFLD (Supplemental Figure 5, J-L).

As described above, the rises in hepatic Tsk expression and circulating TSK were prominent in MCD diet-fed mice (Figure 2, G and L). In order to determine whether TSK loss could affect NAFLD severity in 
A

\begin{tabular}{lcccc} 
& \multicolumn{2}{c}{ Liver triglycerides } & \\
\cline { 2 - 2 } & Low & & High & p value \\
\hline Patient number $(\mathrm{n})$ & 29 & 29 & - \\
\hline Liver triglycerides $(\mathrm{mg} / \mathrm{g}$ liver) & $12.8 \pm 1.3$ & & $38.4 \pm 1.6$ & $<0.0001$ \\
\hline BMI $\left(\mathrm{kg} / \mathrm{m}^{2}\right)$ & $50.3 \pm 2.4$ & & $51.0 \pm 1.8$ & 0.8133 \\
\hline Age (years) & $45.6 \pm 1.7$ & & $42.2 \pm 1.7$ & 0.1664 \\
\hline
\end{tabular}
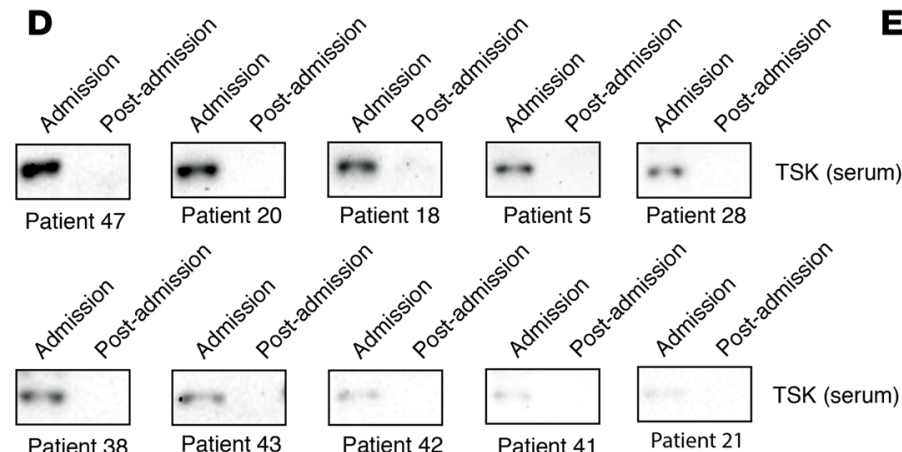

Patient 41

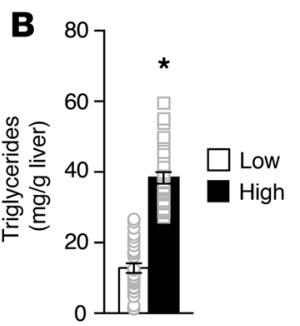

E

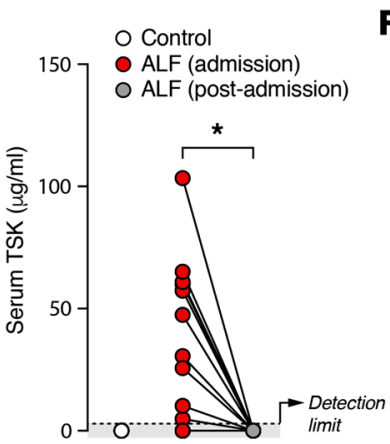

F
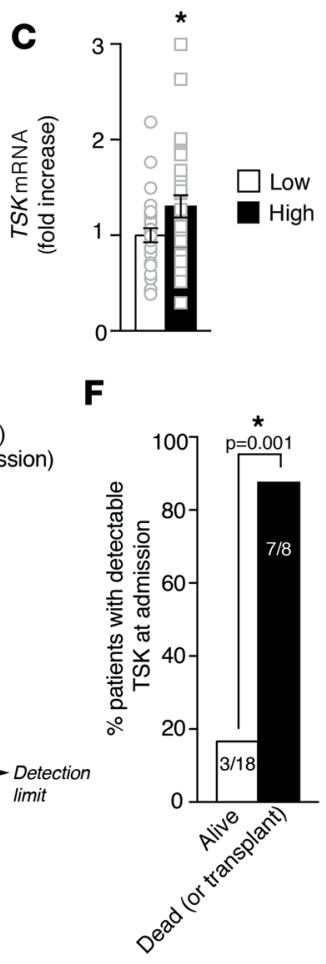

G

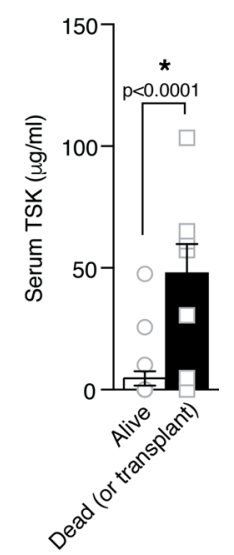

Figure 4. TSK levels are increased in response to steatosis and liver damage in humans. (A) Characteristics of the human patients included in our study. Liver samples were collected from 58 patients and triglycerides were extracted and measured. Patients were distributed into 2 groups based on the median triglyceride content of the samples. (B) Triglyceride content of the liver samples described in $\mathbf{A}$ ( $n=29 / g r o u p)$. (C) TSK mRNA expression levels measured in humans with low or high hepatic triglycerides ( $n=29 /$ group). (D) Western blot analysis and (E) quantification of TSK in serum of humans suffering from acetaminophen-induced ALF. Blood samples were collected from patients upon their admission to the intensive care unit. Other blood samples were collected after admission (between 3 and 9 days) $(n=26)$. The samples presented in $\mathbf{D}$ are the ones in which TSK was detected and quantified. (F) Proportion of ALF patients with detectable serum TSK at admission that either died or received a liver transplant versus the patients that survived. (C) Comparison of TSK levels at admission between patients that either died or received a liver transplant versus the patients that survived. In all panels, data are presented as the mean \pm SEM. In $\mathbf{A}$ and $\mathbf{B},{ }^{*} P<0.05$ vs. controls by 2 -tailed, unpaired $t$ test. In $\mathbf{E},{ }^{*} P<0.05$ comparing TSK at admission vs. after admission by 2 -tailed, paired $t$ test. In $\mathbf{F},{ }^{*} P<0.05$ vs. survivors by Fisher's exact test. $\ln \mathbf{G},{ }^{*} P<0.05$ vs. survivors by 2 -tailed, unpaired $t$ test.

this model, wild-type and TSK-KO mice were fed an MCD diet. Confirming previous observations, MCD diet increased circulating TSK levels in wild-type animals (Supplemental Figure 5M). As shown in Supplemental Figure 5N, we found that wild-type and TSK-KO mice lost the same amount of weight in response to MCD diet. No differences in organ weight were found between the genotypes (Supplemental Figure 5O). Importantly, the loss of TSK did not affect hepatic triglyceride content (Supplemental Figure 5, P and Q). Moreover, we found no effect of TSK loss on the expression of genes regulating inflammation, fibrosis, and ER stress (Supplemental Figure 5R), indicating that TSK loss does not affect the severity of NAFLD in animals fed an MCD diet. Studies were next performed to define whether the loss of TSK could affect liver recovery following a challenge with MCD diet. In this experiment, wild-type and TSK-null mice were fed an MCD diet for 21 days and were next switched back a normal diet for 6 days (Supplemental Figure 5S). As shown in Supplemental Figure 5, T and U, liver triglyceride content and proinflammatory gene expression were not significantly affected by the loss of TSK in this model. Altogether, these results indicate that TSK loss does not affect NAFLD severity and development in various mouse models.

TSK affects systemic cholesterol homeostasis. We next tested the possibility that TSK secretion may impact lipid homeostasis. Plasma triglycerides and NEFA levels were not affected by TSK overexpression (Supplemental Figure 6A). However, we measured a significant decrease in circulating cholesterol levels in these mice (Figure 6A). We found that TSK mostly impacted HDL-cholesterol levels. Of note, the opposite phenotype was observed in TSK-null mice (Supplemental Figure 6B). Supporting these findings, circulating levels of ApoA1, the primary apolipoprotein found in HDL, were reduced in AAV8-TSK but increased in TSK-null mice (Supplemental Figure 6, C and D). Importantly, the impact of TSK on HDL cholesterol was reproduced in LDL receptor-KO (LDLR-KO) mice, indicating that TSK affects plasma cholesterol 
A

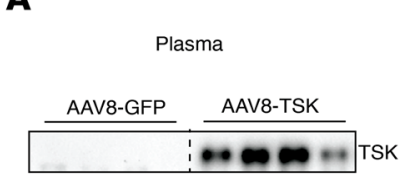

D
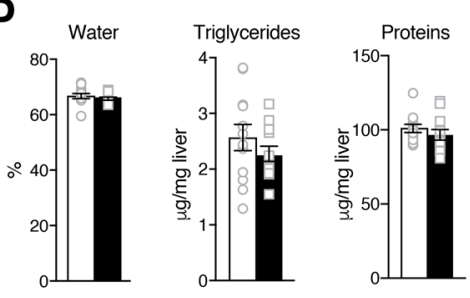

$\mathbf{F}$

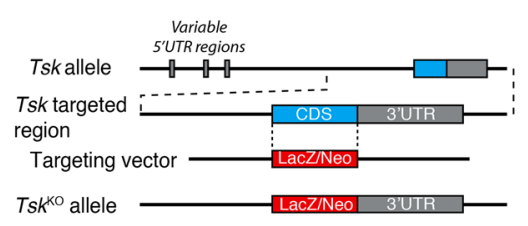

B

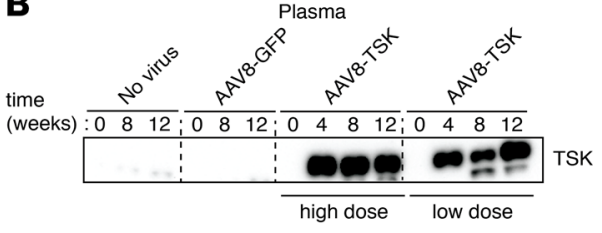

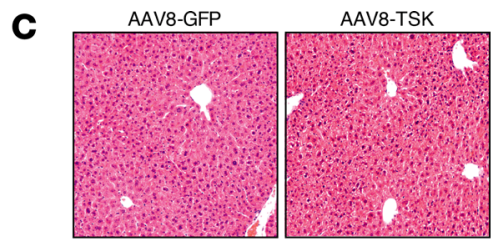

E

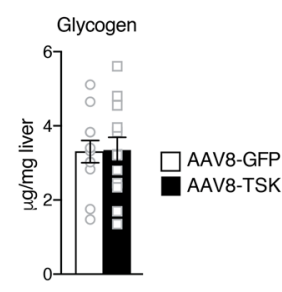

G

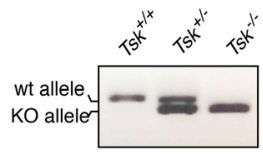

H

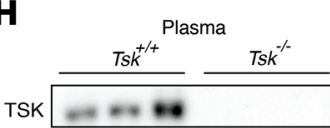

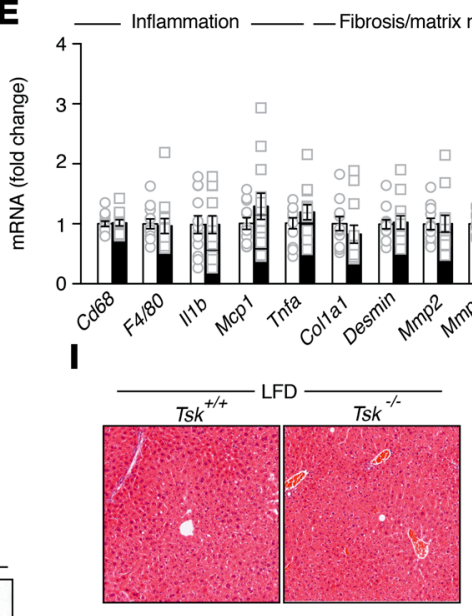

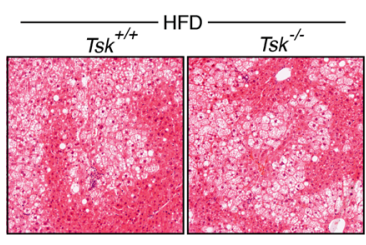

J

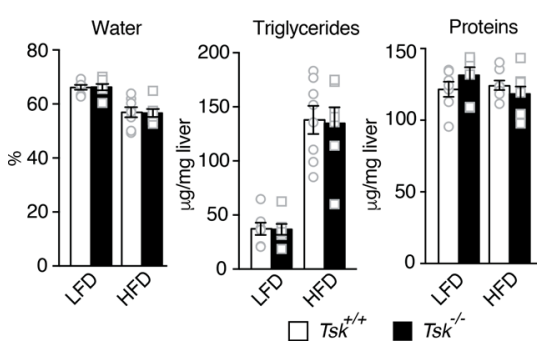

K

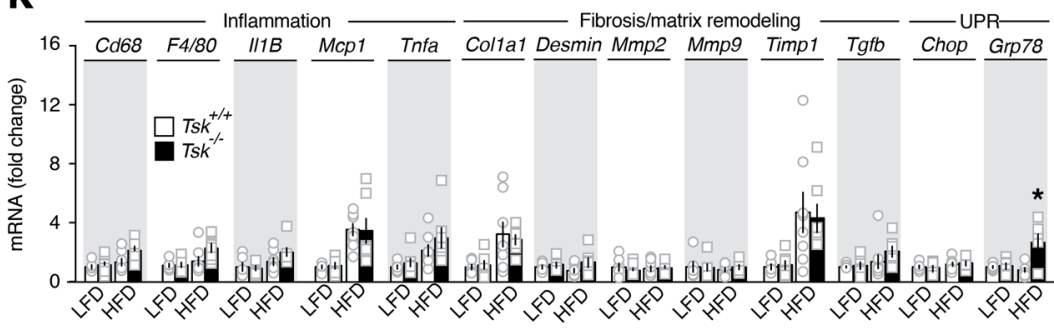

Figure 5. TSK does not affect NAFLD development. (A) Male mice (10 weeks old) were injected with AAV8-GFP or AAV8-TSK. Plasma samples were analyzed by Western blot 4 weeks following AAV8 injection. Representative samples are shown. (B) Western blot showing that mice injected with AAV8-TSK exhibit a stable rise in circulating TSK protein levels. (C) Hematoxylin and eosin-stained (H\&E-stained) sections of liver samples collected from AAV8-GFP and AAV8-TSK mice. Representative images are shown. Original magnification, $\times 10$. (D) Water, triglyceride, protein, and glycogen content of liver samples collected from AAV8-GFP and AAV8-TSK mice ( $n=12$ /group). (E) qPCR analysis of various genes in the liver of AAV8-GFP and AAV8-TSK mice ( $n=11-12$ / group). (F) Schematic overview of the strategy used to produce TSK-KO mice. (G) Genotyping validation showing the wild-type and KO alleles in Tsk ${ }^{+/+}$,

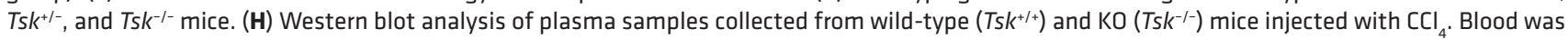

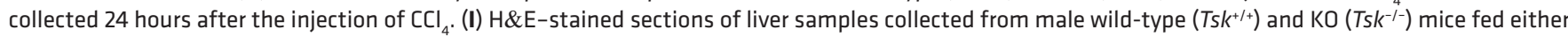
LFD or HFD for 19 weeks. Representative images are shown. (J) Water, triglyceride, protein, and glycogen content of liver samples collected from wild-type

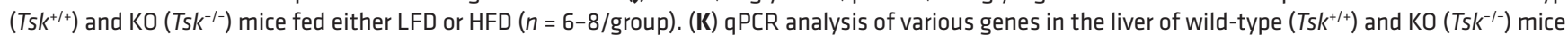
fed either LFD or HFD diet for 19 weeks ( $n=6-8$ /group). In all panels, data are presented as the mean \pm SEM. In $\mathbf{D}$ and $\mathbf{E}$, significance was determined by

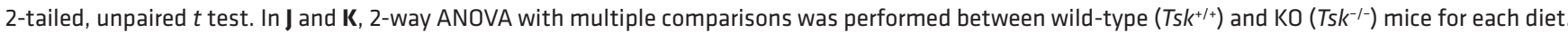
${ }^{*} P<0.05$ versus wild-type $\left(T s \mathrm{k}^{+/+}\right)$mice. Absence of asterisk denotes no significant changes between the groups.

independently of this receptor (Supplemental Figure 6E and Figure 6B). Despite lower HDL cholesterol, we did not find changes in plasma ApoA1 concentration in response to TSK overexpression in this model, indicating that TSK reduces HDL cholesterol independently of ApoA1 modulation in LDLR-KO mice (Supplemental Figure 6F).

HDLs play a central role in reverse cholesterol transport, the critical process of cholesterol movement from the extrahepatic tissues back to the liver. To test whether TSK-mediated changes in HDL levels impact cholesterol efflux, J774 cells were loaded with radiolabeled cholesterol and efflux was measured in the presence of plasma collected from TSK-overexpressing or -KO mice. As shown in Figure 6C, cholesterol efflux was reduced when plasma from AAV8-TSK mice (C57BL6/J or LDLR KO) was used as the cholesterol acceptor. Conversely, a significant rise in cholesterol efflux was measured when plasma from TSK-null 
A

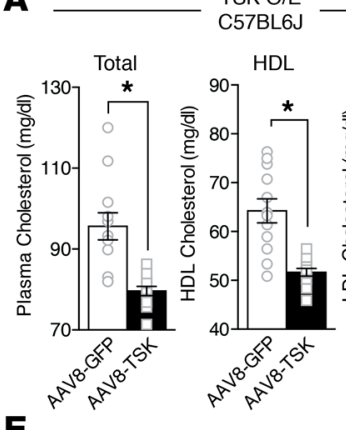

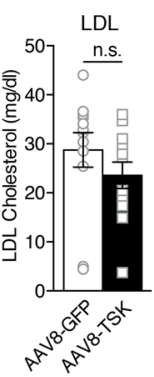

B

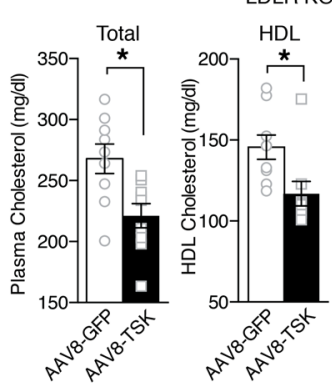

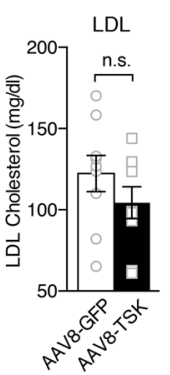

C $-{ }_{\text {C5KBL6J }}^{\text {TSK }-1 \text { TSKO/E }}-$

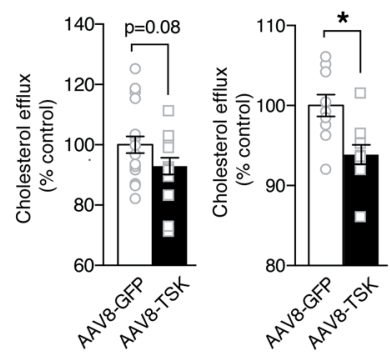

$\mathbf{F}-5$ days -

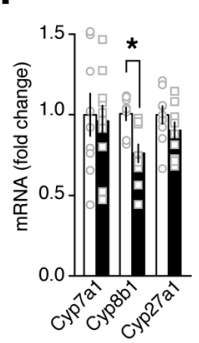

D - TSKKO -

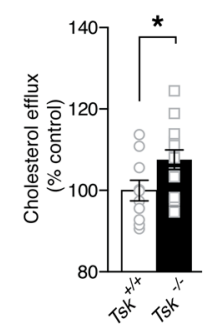

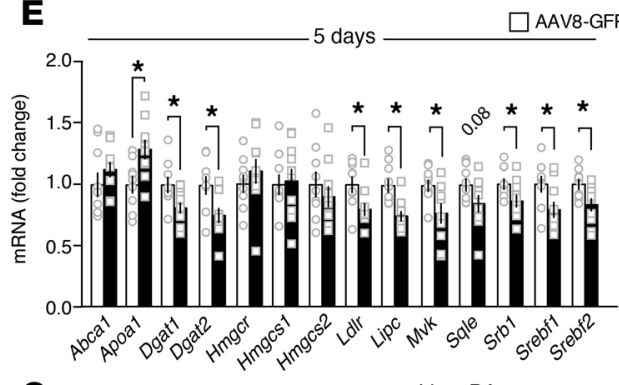

G

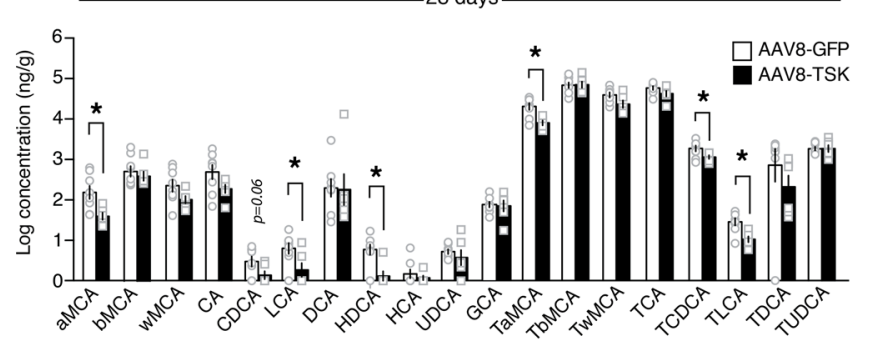

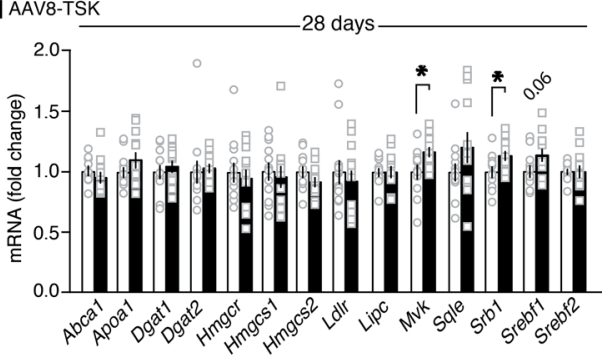

H

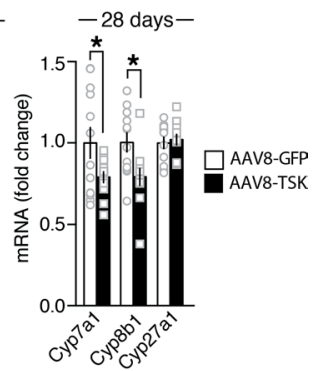

Plasma BAs

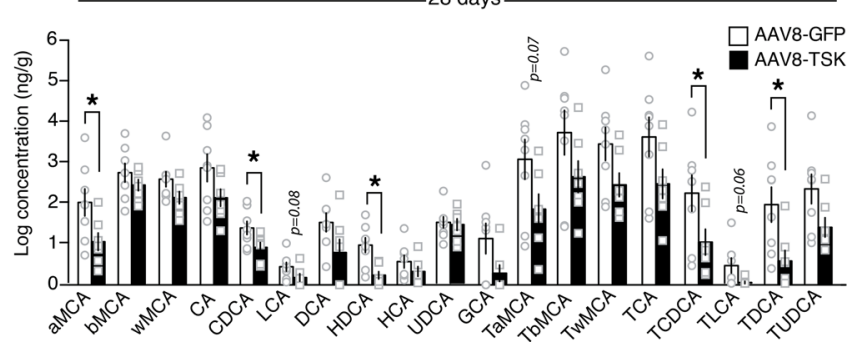

Figure 6. TSK impacts systemic cholesterol homeostasis. (A and B) Plasma cholesterol levels measured in samples collected from male (A) C57BL/6) mice (n $=12$ /group) or (B) LDLR-KO mice ( $n=9$ /group) injected with AAV8-GFP or AAV8-TSK. O/E, overexpression. Blood samples from mice were analyzed 4 weeks following AAV8 injection. (C and D) Cholesterol efflux capacity measured using plasma collected from (C) C57BL/6) mice ( $n=16-19 / g r o u p)$ and LDLR-KO mice ( $n$

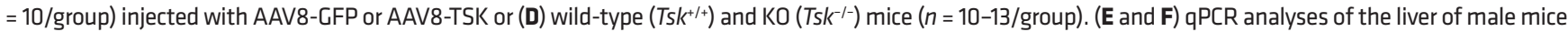
sacrificed after either 5 days (left) or 28 days (right) ( $n=12 /$ group) following injection with AAV8-GFP or AAV8-TSK. (G and H) Bile acid (BA) profiling in (G) the liver and $(\mathbf{H})$ the plasma of mice sacrificed 28 days ( $n=6-8 /$ group) following injection with AAV8-GFP or AAV8-TSK. Values are log transformed. In all panels, data are presented as the mean \pm SEM. Significance was determined by 2 -tailed, unpaired $t$ test. ${ }^{*} P<0.05$ versus control.

mice was used in the assay (Figure 6D). Altogether, these observations indicate that TSK reduces circulating HDL cholesterol, an effect that functionally impacts reverse cholesterol transport.

We next wanted to determine whether TSK affects cholesterol homeostasis by modulating key markers of cholesterol metabolism in the liver. To define how TSK acutely and chronically impacts cholesterol metabolism, mice were sacrificed at early and late time points following AAV8-TSK injection (5 vs. 28 days). AAV8TSK-injected mice showed elevated plasma TSK levels a few days after injection (Supplemental Figure 6G). We found that the reduction in total cholesterol, HDL cholesterol, and circulating ApoA1 was observed rapidly following the injection (Supplemental Figure $6 \mathrm{H}$ ). The expression of genes coding for proteins regulating hepatic cholesterol uptake and synthesis was measured. As shown in Figure 6E, the expression of scavenger receptor B1 (SrbI) and Ldlr, which mediate hepatic cholesterol uptake from HDL and LDL respectively, was reduced by TSK. Despite lower plasma ApoA1 in AAV8-TSK mice, hepatic mRNA expression of Apoa1 was increased in response to TSK, presumably as an attempt to compensate and restore homeostasis. Our analyses next revealed that in addition to reducing markers of cholesterol uptake by the liver, TSK reduced the expression of sterol regulatory element-binding transcription factor 1 (Srebfl) and Srebf2 and some of their target genes (Figure 6E). Altogether, these results indicate that acute TSK expression affects several processes to lower cholesterol flux to the liver. Interestingly, these changes were no longer observed in mice overexpressing TSK chronically for 28 days, even though these animals still showed lower liver cholesterol content, plasma HDL cholesterol, and ApoA1 levels (Figure 6E and Supplemental Figure 6I). 
Reverse cholesterol transport to the liver and its elimination via the biliary pathway represents an important path for cholesterol elimination. We therefore tested whether TSK-mediated reduction in HDL cholesterol and cholesterol efflux translated into changes in cholesterol conversion to BAs in the liver. As shown in Figure 6F, TSK reduced the expression of Cyp $7 a 1$ and Cyp8b1, two rate-limiting enzymes regulating BA synthesis in hepatocytes. This effect was more pronounced at 28 days after injection. Consistent with these findings, targeted metabolomics analyses revealed a significant reduction in several BA species in the liver of AAV8-TSK mice chronically exposed to TSK (Figure 6G and Supplemental Figure 6J). BA profiling in the blood showed a very similar pattern, thus confirming the reduction in BA synthesis (Figure $6 \mathrm{H}$ and Supplemental Figure 6K). Also, AAV8-TSK mice showed a decrease in circulating levels of 7- $\alpha$-hydroxy-4-cholesten-3-one (7aC4), a well-established marker of hepatic BAs synthesis (Supplemental Figure 6L). These findings show that the effect of TSK on circulating HDL levels and cholesterol efflux are associated with a coinciding decrease in cholesterol conversion to BAs in the liver. Collectively, our findings support a model in which TSK impacts several metabolic processes to regulate cholesterol homeostasis.

\section{Discussion}

Here, we report the identification of TSK as a hepatokine expressed and released in response to NAFLD and liver damage in mice and humans. Gain- and loss-of-function studies revealed that TSK impacts cholesterol homeostasis. We report that TSK lowers circulating HDL cholesterol, an effect linked to lower cholesterol efflux capacity and reduced cholesterol-to-BA conversion in the liver. Our data identify the hepatokine TSK as a blood biomarker of liver stress that could link NAFLD to the development of atherogenic dyslipidemia and atherosclerosis.

Inflammation and ER stress play crucial roles in promoting Tsk expression and release by the liver. Our observations indicate that these metabolic stresses, often present in the liver of obese patients (45, 46), could act in concert to trigger the transcription and release of TSK. Here, we found that proinflammatory cytokines (TNF- $\alpha$, IL-1 $\beta$, and IFN- $\gamma$ ) act additively to promote Tsk transcription in hepatocytes. We also observed that ER stress drives Tsk transcription downstream of IRE1A and PERK, two major effectors of the UPR. The precise identity of the transcription factors that activate Tsk transcription remains to be determined. It is relevant to point out that the relationship between hepatic Tsk transcription and TSK protein release in the blood is not linear. For instance, the rise in circulating TSK levels induced by various hepatic stresses was often very robust, even in the face of modest increases in Tsk mRNA expression. This indicates that the elevation in circulating TSK likely involves other points of control, beyond the rise in Tsk transcription. It is possible that hepatic stress could activate Tsk translation, promote its secretion efficiency, and/or increase its half-life in circulation. Additionally, we cannot exclude the possibility that TSK could, as with other members of the SLRP family, accumulate in the extracellular matrix and be released in response to stress $(34,52)$. This mode of action could ensure a fast liberation of TSK to maximize its effects in response to liver injuries. Additional studies are needed to address all these potential mechanisms underlying TSK release in NAFLD.

NAFLD is associated with insulin resistance and atherosclerosis and predicts cardiovascular events in humans (53-58). Atherogenic alterations in lipoprotein subclasses are found in NAFLD, including increased small and dense LDL particles, low HDL cholesterol, low ApoA1, and elevated triglycerides (59-62). Alterations in circulating HDL subfractions have also been detected in patients with NAFLD (60). In line with these findings, reduced cholesterol efflux capacity was detected in plasma from humans with NAFLD $(59,63)$. Such reduction in cholesterol efflux was proposed as a possible mechanism linking NAFLD to cardiovascular diseases $(59,63)$. How exactly NAFLD affects HDLs to reduce reverse cholesterol transport and promote cardiovascular risk is still elusive. Here, we provide evidence that the hepatokine TSK, which is actively secreted in response to NAFLD, controls HDL-cholesterol levels, cholesterol efflux, and BA synthesis in mice. Our observations are in line with other reports showing that inflammation, a condition that increases TSK, also impairs reverse cholesterol transport and BA production in both mice and humans (64). Collectively, our findings suggest that TSK might be part of a biological response aimed at limiting reverse cholesterol transport to the liver when hepatocytes are exposed to stressful conditions. Although the initial rise in TSK may exert beneficial effects for stressed hepatocytes by reducing cholesterol flux to the liver, its sustained elevation in obesity and NAFLD could potentially contribute to atherosclerosis by chronically limiting reverse cholesterol transport. 
How TSK controls cholesterol homeostasis is an important question. Here, we show that hepatic TSK acutely modulates gene expression in the liver, indicating that TSK acts at least via an autocrine and/or paracrine mechanism. This supports previous literature showing that TSK is an extracellular protein that can locally affect the activity of several signaling pathways (51). Studies in chick and Xenopus showed that TSK controls BMP, Wnt, FGF, and TGF- $\beta$ signaling during development and organogenesis by interacting with various soluble factors and receptors (51). The impact of TSK on these signaling cascades in adult tissues has never been investigated to our knowledge. We did not observe effects of TSK on the expression or phosphorylation levels of effectors of these signaling cascades (data not shown). Nevertheless, owing to the pleiotropic nature of TSK, other signaling nodes could likely be targeted by this hepatokine. Also, we cannot exclude the possibility that TSK could directly bind to a still unidentified membrane receptor or even interact with proteins in the plasma to modulate their functions.

Our data are consistent with the very recent identification of TSK as a new hepatokine $(43,65)$. However, our findings differ from that of Xiong et al. who showed that TSK deletion protects mice against NASH pathologies (65). In this report, the authors found that TSK-null mice exhibit lower liver triglycerides, inflammation, and fibrosis versus wild-type littermates (65). Here, neither the loss nor the overexpression of TSK had any effect on NAFLD development and progression. Several factors could have contributed to such phenotypical discordance. The most important difference between our respective reports is related to the use of different protocols to induce NAFLD. Xiong et al. used a special diet containing fructose, fat, and cholesterol for 6 months (65), a regimen known to promote a high degree of liver damage in mice. In contrast, we have tested the impact of TSK loss in HFD-fed mice, ob/ob mice, and MCD diet-fed mice. Although all these conditions promote NAFLD, the intensity of steatosis, inflammation, and fibrosis was probably lower in these models (66). It is possible that the beneficial effect linked to TSK loss could be apparent only in conditions of extreme liver defects. Another possible factor that could have contributed to the different outcomes of our respective reports relates to the obesity susceptibility between the TSK mouse lines used. Here, we found no effect of TSK loss on diet-induced obesity, whereas Xiong et al. showed that TSK deletion prevents obesity $(43,65)$. Owing to the central role of obesity in promoting $\operatorname{NAFLD}(9,10)$, the improvement in liver pathology observed by Xiong et al. could likely be an indirect consequence of reduced body weight in these mice $(43,65)$. The exact reason why our respective TSK-null mouse models showed a different sensitivity to weight gain is unknown. Differences in the genetic background may have contributed to these differences. Indeed, the TSK-KO models used by our respective teams were generated by 2 independent groups that used embryonic stem cells originating from different mouse strains $(67,68)$. Although both KO mice were backcrossed to C57BL/6J for several generations, differences in the genetic background likely persisted between the models, which could have been sufficient to alter the response to TSK loss. The contribution of genetic background to obesity susceptibility and obesity-related metabolic disturbances is well established (69-71). Alternatively, it is possible that variations in housing conditions and gut microbiota may also have affected the biological outcomes of each report (72). Additional studies are needed to resolve these issues.

Here, we provide evidence that TSK is a hepatokine whose expression and circulating levels are induced in response to NAFLD and liver damage not only in mice but also in humans. In a human cohort, we found that TSK expression was significantly higher in patients with elevated hepatic triglyceride content. Unfortunately, circulating TSK remained under the detection limit in these samples. To demonstrate that TSK can be detected in human plasma in response to severe hepatic stress, we took advantage of samples collected from patients suffering from acetaminophen-induced ALF. In this cohort, we found that TSK levels were significantly lower in ALF patients who survived without liver transplant than those that either died or required emergency transplantation. These results suggest that TSK may potentially be used as a biomarker to discriminate between patients with adequate remaining liver parenchyma that may recover from ALF from those that may not. Our findings thus indicate that plasma TSK may have prognostic significance in these patients. Additional studies with a larger population are needed to confirm this possibility.

In conclusion, we report the identification of TSK as a hepatokine produced by the liver in response to NAFLD. We provide evidence that TSK affects cholesterol homeostasis by modulating circulating HDL cholesterol, reverse cholesterol transport, and BA synthesis in the liver. Collectively, our observations indicate that TSK could represent a new biomarker of liver stress linking NAFLD to atherogenic dyslipidemia and atherosclerosis. Additional studies are needed to define the role of TSK in regulating cholesterol metabolism and atherosclerosis development in humans. 


\section{Methods}

Animal care. All mice were on a C57BL/6J background. Wild-type C57BL/6J (stock number 000664), obese (ob/ob; stock number 000632 and $d b / d b$; stock number 000697), and LDLR-KO mice (stock number 002207) were purchased from the Jackson Laboratory. Male mice were used in all the studies. Mice were maintained on a 12-hour light/12-hour dark cycle (lights on 0600-1800 hours) while individually housed in ventilated cages at an ambient temperature of $23.1^{\circ} \mathrm{C}$. Unless stated, all mice were fed ad libitum chow. The low-fat diet (LFD) (10\% kcal from fat, D12450B) and HFD (60\% kcal from fat, D12492) were purchased from Research Diets. The control diet (A02082003B) and MCD (A02082002B) diet were also purchased from Research Diets.

Generation of Tsk-KO mice. Tsk-KO mice were obtained from Genentech/Lexicon and were generated by homologous recombination, as described previously (68). The Tsk wild-type allele was amplified using the following primers: forward 5'-GACATCAATCTGAGCCATAAC-3', reverse 5'-ATGAAGGCATCTGGGTTGATG-3'. The integration of the Neo cassette in the mutant Tsk allele was measured using the following primers: forward 5'-GTAGACTCTCCACAGGCATTGG-3', reverse 5'-GCAGCGCATCGCCTTCTATC-3'. Mice were backcrossed for 6 generations onto C57BL/6J before being used experimentally. All the experiments were performed using age-matched littermates produced from breeding Tsk heterozygous mice.

Overexpression of TSK in mice. The AAV plasmid used to overexpress TSK contains an expression cassette consisting of the CMV enhancer and CBA promoter, WPRE, and SV40 polyA flanked by AAV8 inverted terminal repeats. The Tsk gene was inserted into the multiple cloning sites between the CBA promoter and WPRE sequence. AAV vectors were packaged by the Canadian Neurophotonics Platform (Centre de recherche CERVO, Québec, Canada). Briefly, viral particles were generated from a triple transfection of HEK 293T/17 cells (ATCC, CRL-11268) and collected from the culture media 5 days after transfection. They were concentrated using a tangential flow filtration setup (Vivaflow 50R 100-kDa MWCO, Sartorius) and then purified by iodixanol gradient and ultracentrifugation. Purified particles were collected in suspension buffer (PBS containing $320 \mathrm{mM} \mathrm{NaCl}, 5 \%$ D-sorbitol, and 0.001\% Pluronic F-68) and titrated by qPCR (TaqMan) using ITR-based probe and primers. Physical titer and purity were confirmed by separating identical volumes of AAV in $10 \%$ SDS-PAGE (stain free, Bio-Rad) in Tris-glycine-SDS buffer. Male C57BL/6J mice (10 weeks old) or LDLRKO mice were injected with $100 \mu \mathrm{L}$ of AAV8-GFP or AAV8-TSK via the tail vein $\left(1 \times 10^{11}\right.$ plaque-forming units/mouse). The animals were sacrificed at the times indicated in the figure legends following the injection.

Human liver samples. Liver samples were collected from nonsmoking, obese men undergoing bariatric surgery (biliopancreatic diversion with duodenal switch). All participants provided written, informed consent. Triglycerides were extracted from the samples and the patients were distributed into 2 groups based on their median triglyceride content. RNA was extracted from the same samples and TSK expression was measured by qPCR.

Serum samples from ALF patients. Samples were collected along with corresponding clinical information from patients with ALF. Inclusion criteria were the following: for the purposes of this study, ALF was defined as an internationalized ratio (INR) $\geq 1.5$ and hepatic encephalopathy (HE) within the first 26 weeks of liver disease in a patient with an acute hepatic insult (73). The etiology of ALF for all patients in this analysis was acetaminophen toxicity (APAP-ALF). HE grade was defined by the West Haven Criteria (simplified) as follows: grade 1, any alteration in mentation; grade 2, somnolent or obtunded but easily rousable or presence of asterixis; grade 3, rousable with difficulty; and grade 4, unresponsive to deep pain (74) and age $\geq 18$ years. The exclusion criterion was cirrhosis/acute-on chronic liver failure. Serum samples were collected from 26 patients upon admission to the intensive care unit (early; day 1) and after admission (between day 3 to 9) when available. Clinical, biochemical, and outcome data were collected prospectively and were analyzed for the 26 patients included in this study. Data assessed included demographic (age, race, sex), comorbidities, biochemistry at the time of sample collection (complete blood count, creatinine, transaminases, phosphate, INR, bilirubin, lactate), hepatic coma grade, and requirement for organ support (mechanical ventilation, vasopressors, and renal replacement therapy). TSK levels were measured in plasma samples by Western blotting. Recombinant TSK (R\&D Systems, 3940-TS-050) was used as an internal standard to quantify plasma TSK levels.

Cell culture. AML12 cells (ATCC, CRL-2254) were cultured in a 1:1 mixture of DMEM and Ham's F12 medium with $0.005 \mathrm{mg} / \mathrm{mL}$ insulin, $0.005 \mathrm{mg} / \mathrm{mL}$ transferrin, $5 \mathrm{ng} / \mathrm{mL}$ selenium, $40 \mathrm{ng} / \mathrm{mL}$ dexamethasone, and 10\% FBS. To measure the secretion of TSK, HEK 293T (ATCC, CRL-3216) cells overexpressing TSK were plated 1 day before the experiment. To study the role of the UPR in the regulation of Tsk expression, AML12 cells were cultured as described above. Cells were pretreated with either $100 \mu \mathrm{M} 4 \mathrm{u} 8 \mathrm{C}$ (Cayman Chemical) or $1 \mu \mathrm{M}$ GSK2606414 (Cayman Chemical) prior to being exposed to tunicamycin $(1 \mu \mathrm{g} / \mathrm{mL})$ for 8 hours. The following day, cells were washed with PBS 
and the medium was replaced with fresh DMEM containing 10\% FBS (1 mL/well). Supernatant and protein lysates were collected at $0,6,12,24$, and 48 hours for Western blot analyses. Supernatant was centrifuged for 5 minutes to remove cell debris.

Virus preparation and cell infection. Mouse TSK and $\Delta$-TSK were subcloned into MSCV (gift from Lin He, Addgene plasmid 24828). Viruses were produced using gag/pol and CMV VSV-G as the packaging system. Cells were infected for 24 hours in the presence of $8 \mu \mathrm{g} / \mathrm{mL}$ polybrene. After infection, the cells were dispersed into fresh medium. Cells were selected on subsequent days with $1 \mu \mathrm{g} / \mathrm{mL}$ puromycin.

Quantitative real-time PCR. Total mRNA was isolated from tissues using the RNeasy Lipid Tissue Mini Kit (Qiagen, 74104). Total mRNA was isolated from cells using the E.Z.N.A. Total RNA Kit I (Omega Biotek, R6834-02). The RNA concentrations were estimated from the absorbance at $260 \mathrm{~nm}$. cDNA synthesis was performed using the iScript Advanced cDNA Synthesis Kit for RT-qPCR (Bio-Rad) as described previously (75). mRNA extraction and cDNA synthesis were performed following the manufacturer's instructions. cDNA was diluted in DNase-free water (1:15) before quantification by real-time PCR. mRNA transcript levels were measured in duplicate samples using the CFX96 or CFX384 Touch Real-Time PCR system (Bio-Rad). Chemical detection of the PCR products was achieved with SYBR Green (Bio-Rad, 172 5271) as previously described (75). Gene expression was normalized to the expression level of reference genes (18S, Actb, Arbp, and Gapdh).

Triglyceride extraction and measurement. Lipids were extracted from tissues as described by Folch et al. (76). The extracted triglycerides were suspended in isopropanol and quantified using a commercial assay kit (Thermo Fisher Scientific, TR22421).

Cholesterol efflux. Cholesterol efflux assay was performed as previously described (77). Briefly, J774A. 1 macrophages (ATCC, TIB-67) were grown to $70 \%-75 \%$ confluence in 48 -well plates. Cells were then loaded with ${ }^{3} \mathrm{H}$-cholesterol for 18 hours $(0.5 \mu \mathrm{Ci}$ per well; PerkinElmer $)$ in DMEM containing $1 \%$ FBS. On the next day, cells were washed with warm PBS and exposed to PEG-treated mouse serum $(2 \% \mathrm{v} / \mathrm{v}$ final) for 4 hours. Percentage of cholesterol efflux was then calculated as the ratio supernatant/(supernatant + lysate).

Glycogen extraction and measurement. Glycogen was measured in liver samples as described previously (78).

Plasma metabolite measurement. Blood was collected using syringes conditioned with EDTA. Plasma was stored at $-80^{\circ} \mathrm{C}$ for further biochemical analyses. Plasma metabolites were measured using the following commercial kits: insulin (EMD Millipore, SRI-13K), triglycerides (Thermo Fisher Scientific, TR22421), and NEFAs (Wako, 999-34691, 995-34791, 991-34891, 993-35191, and 276-76491). Cholesterol was measured using a commercial assay kit (Randox laboratories, $\mathrm{CH} 200$ ) according to the manufacturer's instructions. Plasma samples were depleted of LDLs and VLDLs using a PEG solution. Briefly, plasma was incubated with a 20\% PEG6000 solution $\left(20 \%\right.$ [w/v] in $200 \mathrm{mM}$ glycine $/ \mathrm{H}_{2} \mathrm{O}$, $\mathrm{pH} 7.4$ ) at a 5:2 ratio on ice for 20 minutes. The mix was centrifuged 30 minutes at $11,000 \mathrm{~g}$ at $4^{\circ} \mathrm{C}$. The supernatant was used to measure HDL cholesterol with the same commercial assay kit used for total cholesterol. Circulating glucose levels were measured from tail blood using an instant glucometer (Roche, Accu-Chek Performa). Mouse Apoa1 was measured using an ELISA kit (Abcam, ab238260).

Western blotting. All cells were rinsed twice with ice-cold PBS before lysis. Cells were lysed with Triton X-100-containing lysis buffer (50 mM HEPES, pH 7.4, 2 mM EDTA, 10 mM sodium pyrophosphate, $10 \mathrm{mM}$ sodium glycerophosphate, $40 \mathrm{mM} \mathrm{NaCl}, 50 \mathrm{mM} \mathrm{NaF}, 2 \mathrm{mM}$ sodium orthovanadate, $1 \%$ Triton $\mathrm{X}-100$, and 1 tablet of EDTA-free protease inhibitors per $25 \mathrm{~mL}$ ). Tissues were homogenized with the same buffer supplemented with $0.1 \%$ sodium lauryl sulfate and $1 \%$ sodium deoxycholate. Cells and tissues were rotated at $4^{\circ} \mathrm{C}$ for 10 minutes and then the soluble fractions of cell lysates were isolated by centrifugation for 10 minutes in a microcentrifuge. Protein levels were then quantified using Bradford reagents and analyzed by Western blotting. Samples were resolved in 10\% Tris-glycine precast gels (Life Technologies). Proteins were transferred to PVDF membranes blocked in 5\% milk diluted in PBS-Tween and incubated with primary antibody overnight at $4^{\circ} \mathrm{C}$. The following antibodies were used: anti-GRP78 (C50B12, Cell Signaling Technology, dilution 1:1000); anti-AKT (pan) (C67E7, Cell Signaling Technology, dilution 1:1000); anti- $\beta$-actin (4967, Cell Signaling Technology, dilution 1:1000); anti-phospho-PKA substrate (RRXS*/T*) (100G7E) (9624S); anti-tyrosine hydroxylase (NB300-109, Novus, dilution 1:1000), and anti-TSK (Immune Bio Solutions; see below). Secondary antibodies were purchased from Cell Signaling Technology (7076S and 7074S) and Immune Biosolutions (Y00008-HRP) and diluted 1:5000. Amersham ECL Western Blotting Detection Reagent (RPN2106) was used to image the blots. 
Antibody generation. Antibodies were raised against TSK in egg-laying White Leghorn hens through a collaboration with Immune Biosolutions. Animals were injected on day 0 with an emulsified mixture of antigen peptide and Freund's complete adjuvant (Rockland). The immunogen sequence corresponds to amino acids 323-335 of mouse TSK (323-EGAYHRQPGSSPK-335). Hens received 2 booster injections with Freund's incomplete adjuvant (Rockland) on days 21 and 42 . The eggs were collected from day 21 to 63. IgYs were harvested from egg yolks. Briefly, egg yolks were degreased and protein containing antibodies precipitated with ammonium sulfate. TSK peptides were coupled with Sulfhydryl-Reactive Resin (ProteoChem). Specific anti-TSK antibodies were then immunopurified on an immunoaffinity column. The concentrations of immunopurified IgYs were estimated by measuring the absorbance at $280 \mathrm{~nm}$ (an optical density at $280 \mathrm{~nm}$ of 1.35 equals $1 \mathrm{mg}$ of $\mathrm{IgY} / \mathrm{mL}$ ).

$B A$ measurements. Plasma, liver, and fecal BAs were assessed using liquid chromatography coupled to tandem mass spectrometry (LC-MS/MS) with an electrospray interface, as previously described (79). The chromatographic system consisted of an Alliance 2690 HPLC apparatus (Waters), and the MS/MS system was an API3200 mass spectrometer (Applied Biosystems).

Statistics. Values are presented as mean \pm SEM. Statistical analyses were performed using GraphPad Prism. When comparing 2 groups, significance was determined by 2 -tailed, unpaired $t$ test. Experiments with multiple groups were analyzed using 1-way ANOVA with Tukey's multiple-comparisons test. Experiments including multiple groups and factors were analyzed using 2-way ANOVA with Sidak's multiple-comparisons test. Fisher's exact test was used to compare the proportion of ALF patients with detectable serum TSK at admission that either died or received a liver transplant versus the patients that survived. For nonparametric variables, significance was determined using Wilcoxon's rank-sum test.

Study approval. All experimental protocols were approved by the Animal Ethics Committee of Université Laval (CPAUL) and in accordance with the guidelines of the Canadian Council on Animal Care. Human liver samples were obtained from the Biobank of the Institut universitaire de cardiologie et de pneumologie de Québec - Université Laval in compliance with Institutional Review Board-approved management modalities. Samples from ALF patients were collected as part of a study approved by the Health Ethics Review Board (HREB) at the University of Alberta (Pro00052591).

\section{Author contributions}

ML, EC, and MM designed the research studies. MM, EC, MA, AC, EJ, LT, IK, MJB, CR, SML, YG, JT, PLM, and ML conducted experiments and acquired data. MM, EC, TVV, JL, FG, PJ, CJK, OB, MCM, and ML analyzed data. CJK, CFR, and WTF provided reagents. WTF, OB, MCM, and AM provided valuable scientific input during the studies. MM and $\mathrm{ML}$ wrote the manuscript.

\section{Acknowledgments}

The authors are grateful to Yves Deshaies, Émile Levy, Roger McLeod, and Benoît Arsenault for advice and assistance. The authors also acknowledge the invaluable collaboration of the surgery team, bariatric surgeons, and biobank staff of the IUCPQ. This work was supported by grants from the Canadian Institutes of Health Research (CIHR) (271671, 374552, and FDN143247), Les Fonds de recherche du Québec - Santé (FRQS) (24726), Le Réseau de recherche en santé cardiométabolique, diabète et obésité (CMDO), Le Réseau de bio-imagerie du Québec (RBIQ), Diabète Québec, La Fondation de l'Institut universitaire de cardiologie et de pneumologie de Québec - Université Laval (IUCPQ-UL), and Merck Sharpe and Dohme Corp./Faculté de Médecine de l'Université Laval to ML. AC is a CIHR Banting postdoctoral fellow.

Address correspondence to: Mathieu Laplante, 2725 chemin Sainte-Foy, Québec, Qc, Canada, G1V 4 G5. Phone: 418.656 .8711 (ext. 3972); Email: mathieu.laplante@criucpq.ulaval.ca.

1. Flegal KM, Kruszon-Moran D, Carroll MD, Fryar CD, Ogden CL. Trends in obesity among adults in the United States, 2005 to 2014. JAMA. 2016;315(21):2284-2291.

2. Ogden CL, et al. Trends in obesity prevalence among children and adolescents in the United States, 1988-1994 through 20132014. JAMA. 2016;315(21):2292-2299.

3. Ng M, et al. Global, regional, and national prevalence of overweight and obesity in children and adults during 1980-2013: a systematic analysis for the Global Burden of Disease Study 2013. Lancet. 2014;384(9945):766-781. 
4. Després JP, Lemieux I. Abdominal obesity and metabolic syndrome. Nature. 2006;444(7121):881-887.

5. Tchernof A, Després JP. Pathophysiology of human visceral obesity: an update. Physiol Rev. 2013;93(1):359-404.

6. Birkenfeld AL, Shulman GI. Nonalcoholic fatty liver disease, hepatic insulin resistance, and type 2 diabetes. Hepatology. 2014;59(2):713-723.

7. Utzschneider KM, Kahn SE. Review: The role of insulin resistance in nonalcoholic fatty liver disease. J Clin Endocrinol Metab. 2006;91(12):4753-4761.

8. Meex RCR, Watt MJ. Hepatokines: linking nonalcoholic fatty liver disease and insulin resistance. Nat Rev Endocrinol. 2017;13(9):509-520.

9. Younossi Z, et al. Global burden of NAFLD and NASH: trends, predictions, risk factors and prevention. Nat Rev Gastroenterol Hepatol. 2018;15(1):11-20.

10. Younossi ZM, Koenig AB, Abdelatif D, Fazel Y, Henry L, Wymer M. Global epidemiology of nonalcoholic fatty liver disease-Meta-analytic assessment of prevalence, incidence, and outcomes. Hepatology. 2016;64(1):73-84.

11. Leite NC, Salles GF, Araujo AL, Villela-Nogueira CA, Cardoso CR. Prevalence and associated factors of non-alcoholic fatty liver disease in patients with type-2 diabetes mellitus. Liver Int. 2009;29(1):113-119.

12. Ludwig J, Viggiano TR, McGill DB, Oh BJ. Nonalcoholic steatohepatitis: Mayo Clinic experiences with a hitherto unnamed disease. Mayo Clin Proc. 1980;55(7):434-438.

13. Wicklow BA, et al. Metabolic consequences of hepatic steatosis in overweight and obese adolescents. Diabetes Care. 2012;35(4):905-910.

14. Oni ET, et al. A systematic review: burden and severity of subclinical cardiovascular disease among those with nonalcoholic fatty liver; should we care? Atherosclerosis. 2013;230(2):258-267.

15. Bonora E, Targher G. Increased risk of cardiovascular disease and chronic kidney disease in NAFLD. Nat Rev Gastroenterol Hepatol. 2012;9(7):372-381.

16. Stefan N, Häring HU. The role of hepatokines in metabolism. Nat Rev Endocrinol. 2013;9(3):144-152.

17. Stefan N, et al. Alpha2-Heremans-Schmid glycoprotein/fetuin-A is associated with insulin resistance and fat accumulation in the liver in humans. Diabetes Care. 2006;29(4):853-857.

18. Meex RC, et al. Fetuin B is a secreted hepatocyte factor linking steatosis to impaired glucose metabolism. Cell Metab. 2015;22(6):1078-1089.

19. Badman MK, Pissios P, Kennedy AR, Koukos G, Flier JS, Maratos-Flier E. Hepatic fibroblast growth factor 21 is regulated by PPARalpha and is a key mediator of hepatic lipid metabolism in ketotic states. Cell Metab. 2007;5(6):426-437.

20. Wu HT, et al. The role of hepassocin in the development of non-alcoholic fatty liver disease. J Hepatol. 2013;59(5):1065-1072.

21. Hansen J, et al. Exercise induces a marked increase in plasma follistatin: evidence that follistatin is a contraction-induced hepatokine. Endocrinology. 2011;152(1):164-171.

22. Hansen JS, et al. Circulating follistatin is liver-derived and regulated by the glucagon-to-insulin ratio. J Clin Endocrinol Metab. 2016;101(2):550-560.

23. Tao R, et al. Inactivating hepatic follistatin alleviates hyperglycemia. Nat Med. 2018;24(7):1058-1069.

24. Hashimoto O, et al. Activin E controls energy homeostasis in both brown and white adipose tissues as a hepatokine. Cell Rep. 2018;25(5):1193-1203.

25. Sugiyama $\mathrm{M}$, et al. Inhibin $\beta E$ (INHBE) is a possible insulin resistance-associated hepatokine identified by comprehensive gene expression analysis in human liver biopsy samples. PLoS ONE. 2018;13(3):e0194798.

26. Lan F, et al. LECT2 functions as a hepatokine that links obesity to skeletal muscle insulin resistance. Diabetes. 2014;63(5):1649-1664

27. Yang Q, et al. Serum retinol binding protein 4 contributes to insulin resistance in obesity and type 2 diabetes. Nature. 2005;436(7049):356-362.

28. Ma X, Zhou Z, Chen Y, Wu Y, Liu Y. RBP4 functions as a hepatokine in the regulation of glucose metabolism by the circadian clock in mice. Diabetologia. 2016;59(2):354-362.

29. Misu H, et al. A liver-derived secretory protein, selenoprotein P, causes insulin resistance. Cell Metab. 2010;12(5):483-495.

30. Lai KK, Kolippakkam D, Beretta L. Comprehensive and quantitative proteome profiling of the mouse liver and plasma. Hepatology. 2008;47(3):1043-1051.

31. Wu C, Jin X, Tsueng G, Afrasiabi C, Su AI. BioGPS: building your own mash-up of gene annotations and expression profiles. Nucleic Acids Res. 2016;44(D1):D313-D316.

32. Wu C, Macleod I, Su AI. BioGPS and MyGene.info: organizing online, gene-centric information. Nucleic Acids Res. 2013;41(Database issue):D561-D565.

33. Su AI, et al. A gene atlas of the mouse and human protein-encoding transcriptomes. Proc Natl Acad Sci USA. 2004;101(16):60626067.

34. Schaefer L, Iozzo RV. Biological functions of the small leucine-rich proteoglycans: from genetics to signal transduction. $J$ Biol Chem. 2008;283(31):21305-21309.

35. Ohta K, et al. Tsukushi functions as an organizer inducer by inhibition of BMP activity in cooperation with chordin. Dev Cell. 2004;7(3):347-358.

36. Ohta K, Kuriyama S, Okafuji T, Gejima R, Ohnuma S, Tanaka H. Tsukushi cooperates with VG1 to induce primitive streak and Hensen's node formation in the chick embryo. Development. 2006;133(19):3777-3786.

37. Morris SA, Almeida AD, Tanaka H, Ohta K, Ohnuma S. Tsukushi modulates Xnr2, FGF and BMP signaling: regulation of Xenopus germ layer formation. PLoS ONE. 2007;2(10):e1004.

38. Kuriyama S, Lupo G, Ohta K, Ohnuma S, Harris WA, Tanaka H. Tsukushi controls ectodermal patterning and neural crest specification in Xenopus by direct regulation of BMP4 and X-delta-1 activity. Development. 2006;133(1):75-88.

39. Ohta K, et al. Tsukushi functions as a Wnt signaling inhibitor by competing with Wnt $2 \mathrm{~b}$ for binding to transmembrane protein Frizzled4. Proc Natl Acad Sci USA. 2011;108(36):14962-14967.

40. Niimori D, et al. Tsukushi controls the hair cycle by regulating TGF- $\beta 1$ signaling. Dev Biol. 2012;372(1):81-87.

41. Niimori D, Kawano R, Niimori-Kita K, Ihn H, Ohta K. Tsukushi is involved in the wound healing by regulating the expression of cytokines and growth factors. J Cell Commun Signal. 2014;8(3):173-177. 
42. Grefhorst A, et al. Stimulation of lipogenesis by pharmacological activation of the liver X receptor leads to production of large, triglyceride-rich very low density lipoprotein particles. J Biol Chem. 2002;277(37):34182-34190.

43. Wang Q, et al. The hepatokine Tsukushi gates energy expenditure via brown fat sympathetic innervation. Nat Metab. 2019;1(1):251-260.

44. Caron A, Lee S, Elmquist JK, Gautron L. Leptin and brain-adipose crosstalks. Nat Rev Neurosci. 2018;19(3):153-165.

45. Pagliassotti MJ. Endoplasmic reticulum stress in nonalcoholic fatty liver disease. Annu Rev Nutr. 2012;32:17-33.

46. Saltiel AR, Olefsky JM. Inflammatory mechanisms linking obesity and metabolic disease. J Clin Invest. 2017;127(1):1-4.

47. Lee AS. The ER chaperone and signaling regulator GRP78/BiP as a monitor of endoplasmic reticulum stress. Methods. 2005;35(4):373-381.

48. Foufelle F, Fromenty B. Role of endoplasmic reticulum stress in drug-induced toxicity. Pharmacol Res Perspect. 2016;4(1):e00211.

49. Krenkel O, Mossanen JC, Tacke F. Immune mechanisms in acetaminophen-induced acute liver failure. Hepatobiliary Surg Nutr. 2014;3(6):331-343.

50. Yano K, et al. The role of Tsukushi (TSK), a small leucine-rich repeat proteoglycan, in bone growth. Regen Ther. 2017;7:98-107.

51. Ahmad SAI, Anam MB, Ito N, Ohta K. Involvement of Tsukushi in diverse developmental processes. J Cell Commun Signal. 2018;12(1):205-210

52. Merline R, Schaefer RM, Schaefer L. The matricellular functions of small leucine-rich proteoglycans (SLRPs). J Cell Commun Signal. 2009;3(3-4):323-335.

53. Targher G, et al. Prevalence of nonalcoholic fatty liver disease and its association with cardiovascular disease among type 2 diabetic patients. Diabetes Care. 2007;30(5):1212-1218.

54. Targher G, et al. Nonalcoholic fatty liver disease and risk of future cardiovascular events among type 2 diabetic patients. Diabetes. 2005;54(12):3541-3546.

55. Brea A, Mosquera D, Martín E, Arizti A, Cordero JL, Ros E. Nonalcoholic fatty liver disease is associated with carotid atherosclerosis: a case-control study. Arterioscler Thromb Vasc Biol. 2005;25(5):1045-1050.

56. Targher G, et al. Relations between carotid artery wall thickness and liver histology in subjects with nonalcoholic fatty liver disease. Diabetes Care. 2006;29(6):1325-1330.

57. Tolman KG, Fonseca V, Dalpiaz A, Tan MH. Spectrum of liver disease in type 2 diabetes and management of patients with diabetes and liver disease. Diabetes Care. 2007;30(3):734-743.

58. Villanova N, et al. Endothelial dysfunction and cardiovascular risk profile in nonalcoholic fatty liver disease. Hepatology. 2005;42(2):473-480

59. Fadaei R, Poustchi H, Meshkani R, Moradi N, Golmohammadi T, Merat S. Impaired HDL cholesterol efflux capacity in patients with non-alcoholic fatty liver disease is associated with subclinical atherosclerosis. Sci Rep. 2018;8(1):11691.

60. Kantartzis K, et al. Fatty liver is independently associated with alterations in circulating HDL2 and HDL3 subfractions. Diabetes Care. 2008;31(2):366-368.

61. Bril F, et al. Hepatic Steatosis and Insulin Resistance, But Not Steatohepatitis, Promote Atherogenic Dyslipidemia in NAFLD. $J$ Clin Endocrinol Metab. 2016;101(2):644-652.

62. Sonmez A, et al. Low- and high-density lipoprotein subclasses in subjects with nonalcoholic fatty liver disease. J Clin Lipidol. 2015;9(4):576-582.

63. van den Berg EH, Gruppen EG, Ebtehaj S, Bakker SJL, Tietge UJF, Dullaart RPF. Cholesterol efflux capacity is impaired in subjects with an elevated Fatty Liver Index, a proxy of non-alcoholic fatty liver disease. Atherosclerosis. 2018;277:21-27.

64. Annema W, Tietge UJ. Regulation of reverse cholesterol transport - a comprehensive appraisal of available animal studies. Nutr Metab (Lond). 2012;9(1):25.

65. Xiong X, et al. Mapping the molecular signatures of diet-induced NASH and its regulation by the hepatokine Tsukushi. Mol Metab. 2019;20:128-137.

66. Lau JK, Zhang X, Yu J. Animal models of non-alcoholic fatty liver disease: current perspectives and recent advances. $J$ Pathol. 2017;241(1):36-44.

67. Ito A, Shinmyo Y, Abe T, Oshima N, Tanaka H, Ohta K. Tsukushi is required for anterior commissure formation in mouse brain. Biochem Biophys Res Commun. 2010;402(4):813-818

68. Tang T, et al. A mouse knockout library for secreted and transmembrane proteins. Nat Biotechnol. 2010;28(7):749-755

69. Almind K, Kahn CR. Genetic determinants of energy expenditure and insulin resistance in diet-induced obesity in mice. Diabetes. 2004;53(12):3274-3285.

70. West DB, Boozer CN, Moody DL, Atkinson RL. Dietary obesity in nine inbred mouse strains. Am J Physiol. 1992;262(6 Pt 2):R1025-R1032

71. Montgomery MK, et al. Mouse strain-dependent variation in obesity and glucose homeostasis in response to high-fat feeding. Diabetologia. 2013;56(5):1129-1139.

72. Ussar S, et al. Interactions between gut microbiota, host genetics and diet modulate the predisposition to obesity and metabolic syndrome. Cell Metab. 2015;22(3):516-530.

73. O'Grady JG, Schalm SW, Williams R. Acute liver failure: redefining the syndromes. Lancet. 1993;342(8866):273-275.

74. Atterbury CE, Maddrey WC, Conn HO. Neomycin-sorbitol and lactulose in the treatment of acute portal-systemic encephalopathy. A controlled, double-blind clinical trial. Am J Dig Dis. 1978;23(5):398-406

75. Labbé SM, et al. In vivo measurement of energy substrate contribution to cold-induced brown adipose tissue thermogenesis FASEB J. 2015;29(5):2046-2058.

76. Folch J, Lees M, Sloane Stanley GH. A simple method for the isolation and purification of total lipides from animal tissues. $J$ Biol Chem. 1957;226(1):497-509.

77. Jubinville É, et al. Pharmacological activation of liver X receptor during cigarette smoke exposure adversely affects alveolar macrophages and pulmonary surfactant homeostasis. Am J Physiol Lung Cell Mol Physiol. 2019;316(4):L669-L678.

78. Lo S, Russell JC, Taylor AW. Determination of glycogen in small tissue samples. J Appl Physiol. 1970;28(2):234-236.

79. Zhang Y, et al. Bcl2 is a critical regulator of bile acid homeostasis by dictating Shp and lncRNA H19 function. Sci Rep $2016 ; 6: 20559$ 